TRANSACTIONS OF THE

AMERICAN MATHEMATICAL SOCIETY

Volume 348, Number 9, September 1996

\title{
HOMOLOGY AND SOME HOMOTOPY DECOMPOSITIONS FOR THE JAMES FILTRATION ON SPHERES
}

\author{
PAUL SELICK
}

\begin{abstract}
The filtrations on the James construction on spheres, $J_{k}\left(S^{2 n}\right)$, have played a major role in the study of the double suspension $S^{2 n-1} \rightarrow$ $\Omega^{2} S^{2 n+1}$ and have been used to get information about the homotopy groups of spheres and Moore spaces and to construct product decompositions of related spaces. In this paper we calculate $H_{*}\left(\Omega J_{k}\left(S^{2 n}\right) ; \mathbb{Z} / p \mathbb{Z}\right)$ for odd primes $p$. When $k$ has the form $p^{t}-1$, the result is well known, but these are exceptional cases in which the homology has polynomial growth. We find that in general the homology has exponential growth and in some cases also has higher $p$-torsion. The calculations are applied to construct a $p$-local product decomposition of $\Omega J_{k}\left(S^{2 n}\right)$ for $k<p^{2}-p$ which demonstrates a mod $p$ homotopy exponent in these cases.
\end{abstract}

\section{INTRODUCTION}

One of the major problems in homotopy theory is to determine the properties of spaces of the form $\Omega X$, where $X$ is a simply connected finite complex. A dichotomy in the rational homotopy of such spaces has been observed by Felix, Halperin, and Thomas $[\mathrm{FHT}]$, who showed that for such $X$, either $\pi_{n}(X) \otimes \mathbb{Q}$ is 0 for all sufficiently large $n$ or else its dimensions grow exponentially with $n$. Expressed in terms of homology rather than homotopy groups, this translates to saying that either $\operatorname{dim} H_{n}(\Omega X ; \mathbb{Q})$ has polynomial growth or it has exponential growth. They introduced the terminology "elliptic" for the first type of space and "hyperbolic" for the second. Independently, John Moore observed that this distinction between elliptic and hyperbolic appears to be reflected in the properties of the torsion homotopy groups of the space as well. He conjectured that an elliptic complex should have a homotopy exponent for each prime while a hyperbolic complex should not have a homotopy exponent for any prime (cf. [S3]). That is, given a prime $p$, there should exist an $r$ such that $p^{r}\left(p\right.$-torsion $\left.\pi_{n}(X)\right)=0$ for all $n$ if and only if $X$ is elliptic. Given a prime $p$, spaces can be further differentiated according to whether or not the mod- $p$ homology of their loop space has polynomial growth or not. We say that $X$ is mod-p elliptic if $H_{n}(\Omega X ; \mathbb{Z} / p \mathbb{Z})$ has polynomial growth and mod-p hyperbolic otherwise. It is not known whether the mod- $p$ homology of mod- $p$ hyperbolic spaces must have exponential growth. Of course if $X$ is hyperbolic, then it is mod $p$ hyberbolic for each prime $p$, but an elliptic space may be mod $p$ hyperbolic as illustrated by the mod $p$ Moore space $S^{k-1} \cup_{p} e^{k}$ which we will write as $P^{k}(p)$.

Received by the editors July 21, 1994.

1991 Mathematics Subject Classification. Primary 55P99, 55P10.

Research partially supported by a grant from NSERC.

(C)1996 American Mathematical Society 
The Moore conjecture has been verified for only a handful of spaces, and with the exception of the Moore space $P^{k}(p)$ all of the elliptic spaces for which it has been verified for a given prime $p$ are also mod- $p$ elliptic at that prime. One of the purposes of this paper is to demonstrate a mod $p$ homotopy exponent for certain elliptic spaces, many of which are mod- $p$ hyperbolic.

Let $J_{k}(X)$ denote the $k$-th filtration of the James construction, $J(X)$, on $X$, where $X$ is a pointed topological space with basepoint $*$. Explicitly, $J_{k}(X)=$ $X^{k} / \sim$, where

$$
\left(x_{1}, \ldots, x_{j-1}, *, x_{j}, x_{j+1}, \ldots, x_{k-1}\right) \sim\left(x_{1}, \ldots, x_{j-1}, x_{j}, *, x_{j+1}, \ldots, x_{k-1}\right) .
$$

Theorem (James). If $X$ is a connected $C W$ complex, then

1) $J(X) \approx \Omega \Sigma X$

2) $\Sigma J_{k}(X) \approx \bigvee_{j=0}^{k} \Sigma X^{(j)}$.

The spaces $J_{k}\left(S^{2 n}\right)$ have played a major role in the study of the double suspension map $E^{2}: S^{2 n-1} \rightarrow \Omega^{2} S^{2 n+1}$ and in determination of properties of homotopy groups of spheres and Moore spaces including construction of product decompositions of related spaces. After localization at a prime $p$ the most tractible of these spaces are those for $k$ of the form $p^{t}-1$. Many properties of these spaces are known, including the fact that $\Omega J_{p^{t}-1}\left(S^{2 n}\right)$ has a mod- $p$ homotopy exponent for $t \geq 0$. However these are exceptional cases which are $\bmod p$ elliptic.

In the first section of this paper we will calculate the mod $p$ homology of $\Omega J_{k}\left(S^{2 n}\right)$ for odd primes $p$. One of the interesting features of this homology is the appearance of higher $p$-torsion in some cases. As we shall see, although $J_{k}\left(S^{2 n}\right)$ is elliptic for all $k$, for each prime $p$ it is mod- $p$ hyperbolic for "most" $k$. In section 2 we will obtain a homotopy decomposition of $\Omega J_{k}\left(S^{2 n}\right)$ after localization at $p$ in the cases $k<p^{2}-p$ and use it to show that $J_{k}\left(S^{2 n}\right)$ has a mod- $p$ homotopy exponent for such $k$. While analogous decompositions may exist for $k \geq p^{2}-p$ (and some cases will be shown in a followup paper [S4]), the existence of non-trivial Steenrod operations (other than the Bockstein) precludes a decomposition of exactly the same form.

Throughout the rest of this paper, let $p$ be an odd prime. We will be working exclusively with $p$-local spaces and will abuse notation by using $X$ to mean $X_{(p)}$.

Our main decomposition theorem states

Theorem. For $k$ such that $p \leq k<p^{2}-p$ and $k \not \equiv-1(p)$

$$
\begin{aligned}
& \Omega J_{k}\left(S^{2 n}\right) \approx F_{2}(n) \\
& \times \Omega\left(S^{2 n(k+1)-1} \vee \bigvee_{j=0}^{\infty} P^{2 n(q+1) p+j(2 n p-2)-1}(p) \vee \bigvee_{j=0}^{\infty} P^{2 n(k+p+1)+j(2 n p-2)-2}(p)\right)
\end{aligned}
$$

where $F_{2}(n)$ is a mod-p elliptic space introduced in [S2] which is known to have a homotopy exponent.

The cases where $p \leq k<p^{2}-p$ and $k \equiv-1(p)$ are elliptic, and in these cases we get the simpler result 
Theorem. For $k$ such that $p \leq k<p^{2}-p$ and $k \equiv-1(p)$

$$
\Omega J_{k}\left(S^{2 n}\right) \approx F_{2}(n) \times \Omega S^{2 n(k+1)-1} .
$$

\section{Homology of $\Omega J_{k}\left(S^{2 n}\right)$}

In this section we calculate the mod- $p$ homology of $\Omega J_{k}\left(S^{2 n}\right)$ as a Hopf algebra by means of $H_{*}\left(\Omega J_{k}\left(S^{2 n}\right) ; \mathbb{Z} / p \mathbb{Z}\right)=H(A(k, n) ; \mathbb{Z} / p \mathbb{Z})$, where $A(k, n)$ is an Adams-Hilton model for $J_{k}\left(S^{2 n}\right)$.

For a graded set $S$, let $\mathbb{T}\langle S\rangle, \mathbb{S}\langle S\rangle, \mathbb{L}\langle S\rangle$ and $\mathbb{L}_{a b}\langle S\rangle$ denote respectively the tensor algebra, free abelian graded algebra, and the free and free abelian Lie algebras on $S$ with $\mathbb{Z}_{(p)}$ coefficients, and let $\mathbb{T}^{\mathbb{Z} / p \mathbb{Z}}\langle S\rangle, \mathbb{S}^{\mathbb{Z} / p \mathbb{Z}}\langle S\rangle, \mathbb{L}^{\mathbb{Z} / p \mathbb{Z}}\langle S\rangle$ and $\mathbb{L}_{a b}^{\mathbb{Z} / p \mathbb{Z}}\langle S\rangle$ denote their mod- $p$ reductions. Let $\mathcal{U}$ denote the universal enveloping algebra functor. Thus $\mathbb{T}\langle S\rangle=\mathcal{U} \mathbb{L}\langle S\rangle$ and $\mathbb{S}\langle S\rangle=\mathcal{U} \mathbb{L}_{a b}\langle S\rangle$.

The following lemmas are standard.

Lemma 1.1. Let $A=\mathbb{L}\left\langle a_{1}, a_{2}, \ldots\right\rangle$. Let $a_{i}^{\prime}=u_{i} a_{i}+c_{i}$, where $u_{i}$ is a unit in $\mathbb{Z}_{(p)},\left|c_{i}\right|=\left|a_{i}\right|$, and $c_{i} \in[A, A]$. Then the induced Lie algebra homomorphism $\mathbb{L}\left\langle a_{1}^{\prime}, a_{2}^{\prime}, \ldots\right\rangle \rightarrow A$ is an isomorphism. Similarly if $B=\mathbb{L}\left\langle a_{1}, b_{1}, a_{2}, b_{2}, \ldots\right\rangle$ and $a_{i}^{\prime}=u_{i} a_{i}+\lambda_{i} b_{i}+c_{i}$, where $u_{i}$ is a unit in $\mathbb{Z}_{(p)}, \lambda_{i} \in \mathbb{Z}_{(p)},\left|c_{i}\right|=\left|a_{i}\right|$, and $c_{i} \in$ $[A, A]$, then the induced Lie algebra homomorphism $\mathbb{L}\left\langle a_{1}^{\prime}, b_{1}, a_{2}^{\prime}, b_{2}, \ldots\right\rangle \rightarrow B$ is an isomorphism.

Lemma 1.2. Let $\mathbb{L}\langle S\rangle$ be a differential Lie algebra $(D G L)$ and let $A=\mathbb{L}\langle S \cup\{a, b\}\rangle$ where $d a=b$. Then the canonical inclusion $\mathbb{L}\langle S\rangle \rightarrow A$ and surjection $A \rightarrow \mathbb{L}\langle S\rangle$ induce inverse isomorphisms on homology.

It is well known (cf. [A]) that $A(k, n)=\mathcal{U} L_{0}(k, n)$ where

$$
L_{0}(k, n)=\mathbb{L}\left\langle y_{1}, y_{2}, \ldots, y_{k}\right\rangle,
$$

with $\left|y_{m}\right|=2 n m-1$ and

$$
d\left(y_{m}\right)=-\frac{1}{2} \sum_{i=1}^{m-1}\left(\begin{array}{c}
m \\
i
\end{array}\right)\left[y_{i}, y_{m-i}\right]
$$

In the notation for this and other Lie algebras which will be introduced, we will omit $k$ and $n$ when there is no possibility of confusion. Conversely we might write $y_{m, n}$ for additional clarity when more than one $n$ is under consideration. Write $k=c_{0}+c_{1} p+c_{2} p^{2}+\ldots+c_{t} p^{t}$. Set $k_{0}=k, k_{1}=\left(k_{0}-c_{0}\right) / p, k_{2}=\left(k_{1}-c_{1}\right) / p, \ldots$, $k_{t}=c_{t}$.

Let $L_{1}$ denote the DGL kernel of the surjection $L_{0} \rightarrow \mathbb{L}_{a b}\left\langle y_{1}\right\rangle$. Then

$$
L_{1}=\mathbb{L}\left\langle z_{2}, y_{2}, z_{3}, y_{3}, \ldots, z_{k}, y_{k}, z_{k+1}\right\rangle,
$$

where $z_{m}=\left[y_{1}, y_{m-1}\right]$. For degree reasons the algebraic Serre spectral sequence for the short exact sequence of differential Hopf algebras $\mathcal{U} L_{1} \rightarrow \mathcal{U} L_{0} \rightarrow \mathcal{U} \mathbb{L}_{a b}\left\langle y_{1}\right\rangle$ collapses to give

$$
\begin{aligned}
H_{*}\left(\mathcal{U} L_{0}\right) & \cong H_{*}\left(\mathcal{U} L_{1}\right) \otimes H_{*}\left(\mathcal{U} \mathbb{L}_{a b}\left\langle y_{1}\right\rangle\right) \\
& \cong H_{*}\left(\mathcal{U} L_{1}\right) \otimes \mathbb{S}\left\langle y_{1}\right\rangle
\end{aligned}
$$

as coalgebras. 
We wish to change basis for $L_{1}$, using Lemma 1.1, so as to be able to pair off some generators under $d$ and be able to apply Lemma 1.2.

Let $\nu_{p}(m)$ or simply $\nu(m)$ denote the largest integer such that $p^{\nu_{p}(m)}$ divides $m$. For $m \leq k$ let $z_{m}^{\prime}$ be $1 / p^{\nu(m)}$ times the sum of those terms in the defining expression for $d y_{m}$ indexed by integers $j$ such that $j$ and $m-j$ are not both divisible by $p$.

Explicitly,

$$
z_{m}^{\prime}=-\frac{1}{2} \sum_{\substack{j=1, j \neq 0(p) \text { or } m-j \neq 0(p)}}^{m-1} \frac{1}{p^{\nu(m)}}\left(\begin{array}{c}
m \\
j
\end{array}\right)\left[y_{j}, y_{m-j}\right] .
$$

Thus

$$
\begin{aligned}
z_{m}^{\prime} & =-\frac{m}{p^{\nu(m)}} z_{m}-\frac{1}{2} \sum_{\substack{j \neq=2, j \neq 0(p) \text { or } m-j \neq 0(p)}}^{m-2} \frac{1}{p^{\nu(m)}}\left(\begin{array}{c}
m \\
j
\end{array}\right)\left[y_{j}, y_{m-j}\right] \\
& =-\frac{m}{p^{\nu(m)}} z_{m}+\left[L_{1}, L_{1}\right]
\end{aligned}
$$

where here (and elsewhere) $x+[A, B]$ is used to indicate an element congruent to $x$ modulo the subspace generated by $\{[a, b] \mid a \in A, b \in B\}$.

Although there is no $y_{k+1}$ in $L_{1}(k, n)$, the above expression for $z_{m}^{\prime}$ does make sense when $m=k+1$, so we use it to extend the definition of $z_{m}^{\prime}$ to the case $m=k+1$. Since $m / p^{\nu(m)}$ is a unit in $\mathbb{Z}_{(p)}$, we can use Lemma 1.1 to change basis and write

$$
L_{1}=\mathbb{L}\left\langle z_{2}^{\prime}, y_{2}, z_{3}^{\prime}, y_{3}, \ldots, z_{k}^{\prime}, y_{k}, z_{k+1}^{\prime}\right\rangle .
$$

If $m \not \equiv 0(p)$, then all terms in the expression for $d y_{m}$ are terms in $z_{m}^{\prime}$ and so $d y_{m}=z_{m}^{\prime}$ in this case. Notice also that $d y_{p}=p z_{p}^{\prime}$. Let

$$
L_{1}^{\prime}=\mathbb{L}\left\langle z_{p}^{\prime}, y_{p}, z_{2 p}^{\prime}, y_{2 p}, \ldots, z_{k_{1} p}^{\prime}, y_{k_{1} p}, z_{k+1}^{\prime}\right\rangle .
$$

The above definitions imply that $L_{1}^{\prime}$ is closed under the differential and Lemma 1.2 shows that the inclusion of DGL's $L_{1}^{\prime} \hookrightarrow L_{1}$ induces an isomorphism on homology. Let

$$
K_{1}=\mathbb{L}\left\langle z_{p}^{\prime}, y_{p}, z_{2 p}^{\prime}, y_{2 p}, \ldots, z_{k_{1} p}^{\prime}, y_{k_{1} p}\right\rangle .
$$

$K_{1}$ is a sub-Lie algebra of $L_{1}^{\prime}$.

If $k<p$, then $L_{1}^{\prime}=\mathbb{L}\left\langle z_{k+1}^{\prime}\right\rangle$ and so $H_{*}\left(\Omega J_{k}\left(S^{2 n}\right)\right)$ is given by $H_{*}\left(\Omega J_{k}\left(S^{2 n}\right)\right)=$ $\mathbb{S}\left\langle y_{1}, z_{k+1}^{\prime}\right\rangle$. We now suppose that $k \geq p$. Let $L_{2}$ denote the DGL kernel of the surjection $L_{1}^{\prime} \rightarrow \mathbb{L}_{a b}\left\langle z_{p}^{\prime}, y_{p}\right\rangle$. $L_{2}$ becomes a $\mathcal{U} \mathbb{L}_{a b}\left\langle z_{p}^{\prime}, y_{p}\right\rangle$-module under the action induced by $v \cdot w=[v, w]$ for $v \in \mathbb{L}_{a b}\left\langle z_{p}^{\prime}, y_{p}\right\rangle, w \in L_{2}$. Set $a_{m}=\left[y_{p}, y_{m-p}\right]$ and $b_{m}=\left[y_{p}, z_{m-p}^{\prime}\right]$. Then $L_{2}=\mathbb{L}\left\langle B_{2}\right\rangle$, where $B_{2}$ is a basis for the free $\mathcal{U} \mathbb{L}_{a b}\left\langle z_{p}^{\prime}\right\rangle-$ module on

$$
\begin{array}{r}
\left\{b_{2 p}, a_{2 p}, z_{2 p}^{\prime}, y_{2 p}, b_{3 p}, a_{3 p}, z_{3 p}^{\prime}, y_{3 p}, \ldots b_{k_{1} p}, a_{k_{1} p}\right. \\
\left.z_{k_{1} p}^{\prime}, y_{k_{1} p}, z_{k+1}^{\prime}, b_{\left(k_{1}+1\right) p}, a_{\left(k_{1}+1\right) p}, b_{k+p+1}\right\}
\end{array}
$$


After reduction modulo $p$ the algebraic Serre spectral sequence again collapses to give the coalgebra decomposition

$$
H_{*}\left(\mathcal{U} L_{1} ; \mathbb{Z} / p \mathbb{Z}\right) \cong H_{*}\left(\mathcal{U} L_{2} ; \mathbb{Z} / p \mathbb{Z}\right) \otimes \mathbb{S}^{\mathbb{Z} / p \mathbb{Z}}\left\langle z_{p}^{\prime}, y_{p}\right\rangle
$$

so that

$$
H_{*}\left(\Omega J_{k}\left(S^{2 n}\right) ; \mathbb{Z} / p \mathbb{Z}\right) \cong H_{*}\left(\mathcal{U} L_{2} ; \mathbb{Z} / p \mathbb{Z}\right) \otimes \mathbb{S}^{\mathbb{Z} / p \mathbb{Z}}\left\langle y_{1}, z_{p}^{\prime}, y_{p}\right\rangle
$$

as coalgebras.

Our next goal is to rechoose our generators of $L_{2}$ in such a way as to pair off more generators under $d$ and again apply Lemma 1.2 to produce a sub-Lie algebra $L_{2}^{\prime}$ with $L_{2}^{\prime} \hookrightarrow L_{2}$ inducing an isomorphism on homology. We will then show how to relate the homology of $L_{2}^{\prime}$ to that of $L_{1}^{\prime}\left(k_{1}, n p\right)$, thus obtaining the homology of $L_{2}^{\prime}$ in a recursive sense.

For $q$ such that $q \leq k$ and $m$ divisible by $p$ let $a_{q}^{\prime}$ be $1 / p^{\nu(q)-1}$ times the sum of those terms indexed in the defining expression for $d y_{q}$ by integers $j$ such that $j$ and $q-j$ are not both divisible by $p^{2}$. Explicitly,

$$
\begin{aligned}
a_{m p}^{\prime}= & -\frac{1}{2} \sum_{\substack{j=1, j \neq 0(p)}}^{m p-1} \frac{1}{p^{\nu(m p)-1}}\left(\begin{array}{c}
m p \\
j
\end{array}\right)\left[y_{j}, y_{m p-j}\right] \\
& -\frac{1}{2} \sum_{\substack{j=1, j \neq 0(p)}}^{m-1} \frac{1}{\text { or }_{m-j \neq 0(p)}}\left(\begin{array}{c}
m p \\
j p
\end{array}\right)\left[y_{j p}, y_{m p-j p}\right] .
\end{aligned}
$$

Thus

$$
\begin{aligned}
a_{m p}^{\prime}= & p z_{m p}^{\prime}-\frac{1}{p^{\nu(m p)-1}}\left(\begin{array}{c}
m p \\
p
\end{array}\right) a_{m p} \\
& -\frac{1}{2} \sum_{\substack{j=2, j \neq 0(p)}}^{m-2} \frac{1}{p^{\nu(m)-j}-1 \neq 0(p)}\left(\begin{array}{c}
m p \\
j p
\end{array}\right)\left[y_{j p}, y_{m-j p}\right] \\
= & p z_{m p}^{\prime}-\frac{1}{p^{\nu(m p)-1}}\left(\begin{array}{c}
m p \\
p
\end{array}\right) a_{m p}+\left[L_{2}, L_{2}\right] .
\end{aligned}
$$

Note that $\left(1 / p^{\nu(m p)-1}\right)\left(\begin{array}{c}m p \\ p\end{array}\right)$ is a unit modulo $p$ and that if $m \not \equiv 0(p)$, then the definition implies that $d y_{m p}=a_{m p}^{\prime}$.

Differentiating

$$
d y_{m p}=p^{\nu(m p)} z_{m p}^{\prime}-\frac{1}{2} \sum_{i=1}^{m-1}\left(\begin{array}{c}
m p \\
i p
\end{array}\right)\left[y_{i p}, y_{m p-i p}\right]
$$


yields

$$
\begin{aligned}
d z_{m p}^{\prime} & =\frac{1}{2 p^{\nu(m p)}} \sum_{i=1}^{m-1}\left(\begin{array}{c}
m p \\
i p
\end{array}\right) d\left[y_{i p}, y_{m p-i p}\right] \\
& =\frac{1}{p^{\nu(m p)}}\left(\begin{array}{c}
m p \\
p
\end{array}\right) d\left[y_{p}, y_{m p-p}\right]+\frac{1}{2 p^{\nu(m p)}} \sum_{i=2}^{m-2}\left(\begin{array}{c}
m p \\
i p
\end{array}\right) d\left[y_{i p}, y_{m p-i p}\right] \\
& =-\frac{1}{p^{\nu(m p)}}\left(\begin{array}{c}
m p \\
p
\end{array}\right)\left(\left[p z_{p}^{\prime}, y_{m p-p}\right]-\left[y_{p}, d y_{m p-p}\right]\right)+\left[L_{2}, L_{2}\right] \\
& =\frac{1}{p^{\nu(m p)}}\left(\begin{array}{c}
m p \\
p
\end{array}\right)\left[y_{p}, d y_{m p-p}\right]-\frac{p}{p^{\nu(m p)}}\left(\begin{array}{c}
m p \\
p
\end{array}\right)\left[z_{p}^{\prime}, y_{m p-p}\right]+\left[L_{2}, L_{2}\right] .
\end{aligned}
$$

Using the fact that $L_{2}$ is a $\mathcal{U} \mathbb{L}_{a b}\left\langle z_{p}^{\prime}, y_{p}\right\rangle$-module, or directly from the Jacobi identity we see that $\left[y_{p},\left[L_{2}, L_{2}\right]\right] \subset\left[L_{2}, L_{2}\right]$, and so $\left[y_{p},\left[y_{i p}, y_{j p}\right]\right]$ belongs to $\left[L_{2}, L_{2}\right]$ for all $i$ and $j$. Thus

$$
\left[y_{p}, d y_{m p-p}\right]=\left[y_{p}, p^{\nu(m p-p)} z_{m p-p}^{\prime}\right]+\left[L_{2}, L_{2}\right]=p^{\nu(m p-p)} b_{m p}+\left[L_{2}, L_{2}\right] .
$$

Therefore

$$
d z_{m p}^{\prime}=\frac{p^{\nu(m p-p)}}{p^{\nu(m p)}}\left(\begin{array}{c}
m p \\
p
\end{array}\right) b_{m p}-\frac{p}{p^{\nu(m p)}}\left(\begin{array}{c}
m p \\
p
\end{array}\right)\left[z_{p}^{\prime}, y_{m p-p}\right]+\left[L_{2}, L_{2}\right] .
$$

Notice that if $m \not \equiv 1(p)$, then $\left(p^{\nu(m p-p)} / p^{\nu(m p)}\right)\left(\begin{array}{c}m p \\ p\end{array}\right)=\left(p / p^{\nu(m p)}\right)\left(\begin{array}{c}m p \\ p\end{array}\right)$ is a unit modulo $p$.

Turning now to the case $m \equiv 1(p)$, write $m=q p+1$. From (3),

$$
\begin{aligned}
d z_{q p^{2}+p}^{\prime} & =\frac{p^{\nu\left(q p^{2}\right)}}{p^{\nu\left(q p^{2}+p\right)}}\left(\begin{array}{c}
q p^{2}+p \\
p
\end{array}\right) b_{q p^{2}+p}-\frac{p}{p^{\nu\left(q p^{2}+p\right)}}\left(\begin{array}{c}
q p^{2}+p \\
p
\end{array}\right)\left[z_{p}^{\prime}, y_{q p^{2}}\right]+\left[L_{2}, L_{2}\right] \\
& =\frac{p^{\nu\left(q p^{2}\right)}}{p}\left(\begin{array}{c}
q p^{2}+p \\
p
\end{array}\right) b_{q p^{2}+p}-\left(\begin{array}{c}
q p^{2}+p \\
p
\end{array}\right)\left[z_{p}^{\prime}, y_{q p^{2}}\right]+\left[L_{2}, L_{2}\right] .
\end{aligned}
$$

Note that $\left(\begin{array}{c}q p^{2}+p \\ p\end{array}\right)$ is a unit modulo $p$. Our intention is to use these formulas to justify replacing the generators $\left(z_{p}^{\prime}\right)^{j} \cdot b_{m p}$ by $d\left(\left(z_{p}^{\prime}\right)^{j} \cdot d z_{m p}^{\prime}\right)$ for $m \not \equiv 1(p)$ and $j \geq 0$ and replacing $\left(z_{p}^{\prime}\right)^{j} \cdot y_{q p^{2}}$ by $d\left(\left(z_{p}^{\prime}\right)^{j-1} \cdot z_{q p^{2}+p}^{\prime}\right)$, for $j \geq 1$.

Also,

$$
\begin{aligned}
& d b_{q p^{2}+p}=d\left[y_{p}, z_{q p^{2}}^{\prime}\right] \\
& \quad=\left[p z_{p}^{\prime}, z_{q p^{2}}^{\prime}\right]-\left[y_{p}, d z_{q p^{2}}^{\prime}\right] \\
& \quad=p\left[z_{p}^{\prime}, z_{q p^{2}}^{\prime}\right]-\left[y_{p}, \frac{p^{\nu\left(q p^{2}-p\right)}}{p^{\nu\left(q p^{2}\right)}}\left(\begin{array}{c}
q p^{2} \\
p
\end{array}\right) b_{q p^{2}}-\frac{p}{p^{\nu\left(q p^{2}\right)}}\left(\begin{array}{c}
q p^{2} \\
p
\end{array}\right)\left[z_{p}^{\prime}, y_{q p^{2}-p}\right]\right]+\left[L_{2}, L_{2}\right]
\end{aligned}
$$

using the earlier expression for $d z_{q p^{2}}^{\prime}$ and the fact that $\left[y_{p},\left[L_{2}, L_{2}\right]\right] \subset\left[L_{2}, L_{2}\right]$. The Jacobi identity gives $\left[y_{p}, b_{q p^{2}}\right]=\left[y_{p},\left[y_{p}, z_{q p^{2}-p}^{\prime}\right]\right]=(1 / 2)\left[a_{2 p}, z_{q p^{2}-p}^{\prime}\right] \in\left[L_{2}, L_{2}\right]$. Similarly $\left[y_{p},\left[z_{p}^{\prime}, y_{q p^{2}-p}\right]\right]=\left[z_{p}^{\prime}, a_{q p^{2}}\right]+\left[L_{2}, L_{2}\right]$. Therefore

$$
d b_{q p^{2}+p}=p\left[z_{p}^{\prime}, z_{q p^{2}}^{\prime}\right]-\frac{p}{p^{\nu\left(q p^{2}\right)}}\left(\begin{array}{c}
q p^{2} \\
p
\end{array}\right)\left[z_{p}^{\prime}, a_{q p^{2}}\right]+\left[L_{2}, L_{2}\right] .
$$


Noting that $\left(p / p^{\nu\left(q p^{2}\right)}\right)\left(\begin{array}{c}q p^{2} \\ p\end{array}\right)$ is a unit modulo $p$, we shall use this formula to replace $\left(z_{p}^{\prime}\right)^{j} \cdot a_{q p^{2}}$ by $d\left(\left(z_{p}^{\prime}\right)^{j-1} \cdot b_{q p^{2}+p}\right)$ for $j \geq 1$.

Combining (1)-(5) gives

$$
L_{2}=\mathbb{L}\left\langle a_{p^{2}}^{\prime}, y_{p^{2}}, a_{2 p^{2}}^{\prime}, y_{2 p^{2}}, \ldots, a_{k_{2} p^{2}}^{\prime}, y_{k_{2} p^{2}} \cup C_{2} \cup D_{2}\right\rangle
$$

where $C_{2}$ is a basis for the free $\mathcal{U} \mathbb{L}_{a b}\left\langle z_{p}^{\prime}\right\rangle$-module on

$$
\left\{\left\{d y_{m p}, y_{m p}\right\}_{2 \leq m \leq k_{1}, m \neq 0(p)} \cup\left\{d z_{m p}^{\prime}, z_{m p}^{\prime}\right\}_{2 \leq m \leq k_{1}} \cup\left\{d b_{q p^{2}+p}, b_{q p^{2}+p}\right\}_{1 \leq q \leq k_{2}}\right\}
$$

and $D_{2}$ is a basis for the free $\mathcal{U} \mathbb{L}_{a b}\left\langle z_{p}^{\prime}\right\rangle$-module on

$$
\left\{z_{k+1}^{\prime}, c_{\left(k_{1}+1\right) p}, a_{\left(k_{1}+1\right) p}, b_{k+p+1}\right\}
$$

with

$$
c_{\left(k_{1}+1\right) p}= \begin{cases}b_{\left(k_{1}+1\right) p}, & \text { if } k_{1} \neq \equiv(p) \\ z_{p}^{\prime} \cdot y_{k_{1} p}, & \text { if } k_{1} \equiv 0(p) .\end{cases}
$$

Let

$$
K_{2}=\mathbb{L}\left\langle a_{p^{2}}^{\prime}, y_{p^{2}}, a_{2 p^{2}}^{\prime}, y_{2 p^{2}}, \ldots a_{k_{2} p^{2}}^{\prime}, y_{k_{2} p^{2}}\right\rangle,
$$

and let $I_{2}$ be the Lie ideal of $L_{2}$ generated by $C_{2}$.

\section{Lemma 1.3.}

1) $\left[y_{p}, K_{2}\right] \subset I_{2}$.

2) $\left[z_{p}^{\prime}, K_{2}\right] \subset I_{2}$.

Proof. 1) Examination of (2) shows that the terms appearing in that formula as $\left[L_{2}, L_{2}\right]$ always lie in $I_{2}$ and in fact lie in $\left[I_{2}, I_{2}\right]$ when $m \equiv 0(p)$. The Jacobi identity implies that $\left[y_{p},\left[I_{2}, I_{2}\right]\right] \subset I_{2}$ and so

$$
\begin{aligned}
& \equiv\left[y_{p}, p z_{q p^{2}}^{\prime}-\frac{1}{p^{\nu\left(q p^{2}\right)-1}}\left(\begin{array}{c}
q p^{2} \\
p
\end{array}\right) a_{q p^{2}}\right] \\
& =p\left[b_{q p^{2}+p}\right]-\frac{1}{p^{\nu\left(q p^{2}\right)-1}}\left(\begin{array}{c}
q p^{2} \\
p
\end{array}\right)\left[y_{p},\left[y_{p}, y_{q p^{2}-p}\right]\right] \\
& \equiv \frac{1}{2 p^{\nu\left(q p^{2}\right)-1}}\left(\begin{array}{c}
q p^{2} \\
p
\end{array}\right)\left[y_{q p^{2}-p}, a_{2 p}\right] \\
& \equiv 0 \quad \bmod I_{2} .
\end{aligned}
$$

Also

$$
\begin{aligned}
\left(\begin{array}{c}
q p^{2}+p \\
p
\end{array}\right)\left[y_{p}, y_{q p^{2}}\right] & =\left(\begin{array}{c}
q p^{2}+p \\
p
\end{array}\right) a_{q p^{2}+p} \\
& \equiv-a_{q p^{2}+p}^{\prime}+p z_{q p^{2}+p}^{\prime} \\
& \equiv-d y_{q p^{2}+p}+p z_{q p^{2}+p}^{\prime} \\
& \equiv 0 \quad \bmod I_{2}
\end{aligned}
$$

and so $\left[y_{p}, y_{q p^{2}}\right] \in I_{2}$.

2) Let $x$ belong to $K_{2}$. Since $I_{2}$ is a differential ideal $\left(d I_{2} \subset I_{2}\right)$ and $\left[y_{p}, x\right] \in I_{2}$ by (1), differentiating gives $p\left[z_{p}^{\prime}, x\right]-\left[y_{p}, d x\right] \in I_{2}$. Since $\left[y_{p}, d x\right] \in\left[y_{p}, K_{2}\right] \subset I_{2}$, this gives $\left[z_{p}^{\prime}, x\right] \in I_{2}$. 
Since $I_{2}$ is acylic, the preceding lemma implies that the inclusions $\left[y_{p}, K_{2}\right] \hookrightarrow L_{2}$ and $\left[z_{p}, K_{2}\right] \hookrightarrow L_{2}$ induce the zero map on homology. The key step in the recursion is showing that $K_{2}(k, n) \cong K_{1}\left(k_{1}, n p\right)$.

Write $y_{m}^{\#}$ and $z_{m}^{\prime \#}$ for the generators $y_{m, n p}$ and $z_{m, n p}^{\prime}$ of $K_{1}\left(k_{1}, n p\right)$. Let

$$
u(m)=\frac{m !(p !)^{m}}{(m p) !} .
$$

The following lemma is easily checked.

\section{Lemma 1.4.}

a) $u(m)$ is a unit modulo $p$ for all $m$.

b) $\left(\begin{array}{c}m p \\ j p\end{array}\right) u(m)=\left(\begin{array}{c}m \\ j\end{array}\right) u(j) u(m-j)$ for all $j$ and $m$.

Insight into the definition of $u(m)$ is as follows. Let $H: \Omega J\left(S^{2 n}\right) \rightarrow \Omega J\left(S^{2 n p}\right)$ denote the $p$ th Hopf-invariant map. Toda [T] checked that for all $m, H$ induces an isomorphism on $H_{2 n m}\left(; \mathbb{Z}_{(p)}\right)$ by showing that it induces multiplication by $1 / u(m)$ in degree $2 \mathrm{~nm}$.

Define $g: K_{1}\left(k_{1}, n p\right) \rightarrow K_{2}(k, n)$ by $g\left(y_{m p}^{\#}\right)=u(m p) y_{m p^{2}}$ and $g\left(z_{m p}^{\prime \#}\right)=$ $u(m p) a_{m p^{2}}^{\prime}$.

Theorem 1.5. $g$ is an isomorphism of differential graded Lie algebras.

Proof. It is clear that $g$ is an isomorphism of graded Lie algebras, but we must check that $g$ commutes with the differentials.

$$
d^{\#}\left(y_{m p}^{\#}\right)=p^{\nu(m p)} z_{m p}^{\prime \#}-\frac{1}{2} \sum_{j=1}^{m-1}\left(\begin{array}{c}
m p \\
j p
\end{array}\right)\left[y_{j p}^{\#}, y_{m p-j p}^{\#}\right]
$$

and

$$
\begin{aligned}
d\left(y_{m p^{2}}\right) & =p^{\nu\left(m p^{2}\right)-1} a_{m p^{2}}^{\prime}-\frac{1}{2} \sum_{j=1}^{m-1}\left(\begin{array}{c}
m p^{2} \\
j p^{2}
\end{array}\right)\left[y_{j p^{2}}, y_{m p^{2}-j p^{2}}\right] \\
& =p^{\nu(m p)} a_{m p^{2}}^{\prime}-\frac{1}{2} \sum_{j=1}^{m-1}\left(\begin{array}{c}
m p^{2} \\
j p^{2}
\end{array}\right)\left[y_{j p^{2}}, y_{m p^{2}-j p^{2}}\right] .
\end{aligned}
$$

Therefore

$$
\begin{aligned}
g d^{\#}\left(y_{m p}^{\#}\right) & =p^{\nu(m p)} g\left(z_{m p}^{\prime \#}\right)-\frac{1}{2} \sum_{j=1}^{m-1}\left(\begin{array}{c}
m p \\
j p
\end{array}\right)\left[g\left(y_{j p}^{\#}\right), g\left(y_{m p-j p}^{\#}\right)\right] \\
& =u(m p) p^{\nu(m p)} a_{m p^{2}}^{\prime}-\frac{1}{2} \sum_{j=1}^{m-1} u(j p) u((m-j) p)\left(\begin{array}{c}
m p \\
j p
\end{array}\right)\left[y_{j p^{2}}, y_{m p^{2}-j p^{2}}\right] \\
& =u(m p) p^{\nu(m p)} a_{m p^{2}}^{\prime}-u(m p) \frac{1}{2} \sum_{j=1}^{m-1}\left(\begin{array}{c}
m p^{2} \\
j p^{2}
\end{array}\right)\left[y_{j p^{2}}, y_{m p^{2}-j p^{2}}\right] \\
& =u(m p) d y_{m p^{2}} \\
& =d g\left(y_{m p}^{\#}\right)
\end{aligned}
$$


Differentiating (6) and solving for $d^{\#}\left(z_{m p}^{\prime \#}\right)$ gives

$$
p^{\nu(m p)} d^{\#}\left(z_{m p}^{\prime \#}\right)=\frac{1}{2} \sum_{j=1}^{m-1}\left(\begin{array}{c}
m p \\
j p
\end{array}\right) d^{\#}\left(\left[y_{j p}^{\#}, y_{m p-j p}^{\#}\right]\right)
$$

and (7) gives a similar expression for $d\left(a_{m p^{2}}^{\prime}\right)$. Therefore

$$
\begin{aligned}
p^{\nu(m p)} g d^{\#}\left(z_{m p}^{\prime \#}\right) & =\frac{1}{2} \sum_{j=1}^{m-1}\left(\begin{array}{c}
m p \\
j p
\end{array}\right) g d^{\#}\left(\left[y_{j p}^{\#}, y_{m p-j p}^{\#}\right]\right) \\
& =\frac{1}{2} \sum_{j=1}^{m-1}\left(\begin{array}{c}
m p \\
j p
\end{array}\right) d^{\#} g\left(\left[y_{j p}^{\#}, y_{m p-j p}^{\#}\right]\right) \\
& =\frac{1}{2} \sum_{j=1}^{m-1}\left(\begin{array}{c}
m p \\
j p
\end{array}\right) u(j p) u((m-j) p) d\left(\left[y_{j p^{2}}, y_{\left.m p^{2}-j p^{2}\right]}\right]\right) \\
& =\frac{1}{2} \sum_{j=1}^{m-1}\left(\begin{array}{c}
m p^{2} \\
j p^{2}
\end{array}\right) u(m p) d\left(\left[y_{j p^{2}}, y_{\left.\left.m p^{2}-j p^{2}\right]\right)}\right.\right. \\
& =u(m p) p^{\nu(m p)} a_{m p^{2}} \\
& =p^{\nu(m p)} d g\left(z_{m p}^{\prime \#}\right)
\end{aligned}
$$

where we have made use of the previously verified result that $g d^{\#}=d g$ on $y_{m p}^{\#}$. Therefore $g d^{\#}=d g$ on all of the Lie algebra generators.

Remark. Although the situation is not exactly identical, this calculation is essentially the same as that in [A, proof of Lemma 2.6b].

We continue choosing our new generators for $L_{2}$. Let $h=g^{-1}: K_{2}(k, n) \rightarrow$ $K_{1}\left(k_{1}, n p\right)$. We next choose $c_{\left(k_{1}+1\right) p}^{\prime}$ and $a_{\left(k_{1}+1\right) p}^{\prime}$ to replace $c_{\left(k_{1}+1\right) p}$ and $a_{\left(k_{1}+1\right) p}$ and extend the domain of $h: K_{2}(k, n) \rightarrow K_{1}\left(k_{1}, n p\right) \hookrightarrow L_{1}\left(k_{1}, n p\right)$ to include $z_{k+1}^{\prime}$ and these elements.

The definition of $a_{m p}^{\prime}$ we used when $m p \leq k$ will not make sense when $m=k_{1}+1$ since there does not exist $y_{\left(k_{1}+1\right) p}$ in $L_{2}(k, n)$. However there is such an element in $L_{2}(\infty, n)$, and we will denote it as $\hat{y}_{\left(k_{1}+1\right) p}$. In $L_{2}(\infty, n)$ write

$$
d \hat{y}_{\left(k_{1}+1\right) p}=p^{t} \hat{z}_{\left(k_{1}+1\right) p}+p^{t-1} \alpha+\beta,
$$

where $t=\nu\left(\left(k_{1}+1\right) p\right)$,

$$
\begin{gathered}
\hat{z}_{\left(k_{1}+1\right) p}=-\frac{1}{2} \sum_{\substack{j=1, j \neq 0(p)}}^{m-1} \frac{1}{p^{t}}\left(\begin{array}{c}
\left(k_{1}+1\right) p \\
j
\end{array}\right)\left[y_{j}, y_{\left.\left(k_{1}+1\right) p-j\right]}\right] \\
\alpha=-\frac{1}{2} \sum_{\substack{j=1, j \neq 0(p) \text { or } k_{1}+1-j \neq 0(p)}}^{k_{1}} \frac{1}{p^{t-1}}\left(\begin{array}{c}
\left(k_{1}+1\right) p \\
j p
\end{array}\right)\left[y_{j p}, y_{\left(k_{1}+1\right) p-j p}\right],
\end{gathered}
$$


and $\beta$ is the sum of the remaining terms. If $k_{1} \not \equiv-1(p)$, then $t=1$ and $\beta=0$. If $k_{1} \equiv-1(p)$, then $k_{1}+1=\left(k_{2}+1\right) p$ and

$$
\beta=-\frac{1}{2} \sum_{j=1}^{k_{2}}\left(\begin{array}{c}
\left(k_{1}+1\right) p \\
j p^{2}
\end{array}\right)\left[y_{j p^{2}}, y_{\left(k_{1}+1\right) p-j p^{2}}\right] .
$$

Notice that $\alpha$ and $\beta$ lie in $L_{2}(k, n)$. Since differentiating gives

$$
0=p^{t} d \hat{z}_{\left(k_{1}+1\right) p}+p^{t-1} d \alpha+d \beta,
$$

we see that $d \beta$ is divisible by $p^{t-1}$ and $d \alpha+d \beta / p^{t-1}$ is divisible by $p$.

Lemma 1.6. $d \beta=\frac{p^{t-1}}{u\left(\left(k_{1}+1\right) p\right)} g d z_{k_{1}+1}^{\prime \#}$.

Proof. If $k_{1} \not \equiv-1(p)$, then both sides are zero. For $k_{1} \equiv-1(p)$,

$$
d y_{k_{1}+1}^{\#}=p^{t-1}{z^{\prime}}_{k_{1}+1}^{\#}+\frac{1}{2} \sum_{j=1}^{k_{2}}\left(\begin{array}{c}
k_{1}+1 \\
j p
\end{array}\right)\left[y_{j p}^{\#}, y_{k_{1}+1-j p}^{\#}\right]
$$

and so

$$
\begin{aligned}
p^{t-1} g d z_{k_{1}+1}^{\prime \#} & =-\frac{1}{2} \sum_{j=1}^{k_{2}}\left(\begin{array}{c}
k_{1}+1 \\
j p
\end{array}\right) u(j p) u\left(\left(k_{1}+1\right) p-j p\right) d\left[y_{j p^{2}}, y_{\left(k_{1}+1\right) p-j p^{2}}\right] \\
& =-\frac{1}{2} \sum_{j=1}^{k_{2}}\left(\begin{array}{c}
\left(k_{1}+1\right) p \\
j p^{2}
\end{array}\right) u\left(\left(k_{1}+1\right) p\right) d\left[y_{j p^{2}}, y_{\left(k_{1}+1\right) p-j p^{2}}\right] \\
& =u\left(\left(k_{1}+1\right) p\right) d \beta
\end{aligned}
$$

Set $a_{\left(k_{1}+1\right) p}^{\prime}=-\alpha$ and $c_{\left(k_{1}+1\right) p}^{\prime}=d \hat{z}_{\left(k_{1}+1\right) p}=-\left(d \alpha+d \beta / p^{t-1}\right) / p$. Thus

$$
d a_{\left(k_{1}+1\right) p}^{\prime}=p c_{\left(k_{1}+1\right) p}^{\prime}+\frac{g d z_{k_{1}+1}^{\prime \#}}{u\left(\left(k_{1}+1\right) p\right)} .
$$

From the definition,

$$
a_{\left(k_{1}+1\right) p}^{\prime}=\alpha=-\frac{1}{p^{t-1}}\left(\begin{array}{c}
\left(k_{1}+1\right) p \\
p
\end{array}\right) a_{\left(k_{1}+1\right) p}+\left[L_{2}, L_{2}\right]
$$

and note that $\left(1 / p^{t-1}\right)\left(\begin{array}{c}\left(k_{1}+1\right) p \\ p\end{array}\right)$ is a unit modulo $p$. Therefore we may replace $a_{\left(k_{1}+1\right) p}$ by $a_{\left(k_{1}+1\right) p}^{\prime}$ in our basis for $L_{2}$. Differentiating gives

$$
p c_{\left(k_{1}+1\right) p}^{\prime}+\frac{g d z_{k_{1}+1}^{\#}}{u\left(\left(k_{1}+1\right) p\right)}=-\frac{1}{p^{t-1}}\left(\begin{array}{c}
\left(k_{1}+1\right) p \\
p
\end{array}\right) d a_{\left(k_{1}+1\right) p}+\left[L_{2}, L_{2}\right] .
$$


Since $g d z^{\prime \prime \#}{ }_{k_{1}+1}^{\#} \in\left[L_{2}, L_{2}\right]$,

$$
\begin{aligned}
c_{\left(k_{1}+1\right) p}^{\prime} & =-\frac{1}{p^{t}}\left(\begin{array}{c}
\left(k_{1}+1\right) p \\
p
\end{array}\right)\left(\left[p z_{p}^{\prime}, y_{k_{1} p}\right]-\left[y_{p}, d y_{k_{1} p}\right]\right)+\left[L_{2}, L_{2}\right] \\
& =-\frac{1}{p^{t}}\left(\begin{array}{c}
\left(k_{1}+1\right) p \\
p
\end{array}\right)\left(\left[p z_{p}^{\prime}, y_{k_{1} p}\right]-\left[y_{p}, p^{\nu\left(k_{1} p\right)} z_{k_{1} p}^{\prime}\right]\right)+\left[L_{2}, L_{2}\right] \\
& =-\frac{1}{p^{t-1}}\left(\begin{array}{c}
\left(k_{1}+1\right) p \\
p
\end{array}\right)\left(\left[z_{p}^{\prime}, y_{k_{1} p}\right]-p^{\nu\left(k_{1}\right)} b_{\left(k_{1}+1\right) p}\right)+\left[L_{2}, L_{2}\right] .
\end{aligned}
$$

Note that $\left[z_{p}^{\prime}, y_{k_{1} p}\right]$ appears in our basis when $k_{1} \not \equiv 0(p)$, so this formula together with the fact that $\left(1 / p^{t-1}\right)\left(\begin{array}{c}\left(k_{1}+1\right) p \\ p\end{array}\right)$ is a unit modulo $p$ justifies the replacement of $c_{\left(k_{1}+1\right) p}$ by $c_{\left(k_{1}+1\right) p}^{\prime}$ in our basis for $L_{2}$ both in the case where $k_{1} \equiv 0(p)$ and the case $k_{1} \not \equiv 0(p)$.

Let

$$
M_{2}=\mathbb{L}\left\langle a_{p^{2}}^{\prime}, y_{p^{2}}, a_{2 p^{2}}^{\prime}, y_{2 p^{2}}, \ldots, a_{k_{2} p^{2}}^{\prime}, y_{k_{2} p^{2}}, z_{k+1}^{\prime}, c_{\left(k_{1}+1\right) p}^{\prime}, a_{\left(k_{1}+1\right) p}^{\prime}\right\rangle .
$$

If $k \not \equiv-1(p)$, then $d z_{k+1}^{\prime}=0$. In the case $k \equiv-1(p)$, we have $k+1=\left(k_{1}+1\right) p$, so this is the one case where $\hat{z}_{\left(k_{1}+1\right) p}$ lies in $L_{2}(k, n)$. In this case $\hat{z}_{\left(k_{1}+1\right) p}=z_{\left(k_{1}+1\right) p}^{\prime}$ and so $d z_{\left(k_{1}+1\right) p}^{\prime}=c_{\left(k_{1}+1\right) p}^{\prime}$. Extend the definition of $h$ to $\bar{h}: M_{2}(k, n) \rightarrow L_{1}^{\prime}\left(k_{1}, n p\right)$ by setting $\bar{h}\left(z_{k+1}^{\prime}\right)=0, \bar{h}\left(c_{\left(k_{1}+1\right) p}^{\prime}\right)=0$, and $\bar{h}\left(a_{\left(k_{1}+1\right) p}^{\prime}\right)=\left(1 / u\left(\left(k_{1}+1\right) p\right)\right) z_{k_{1}+1}^{\prime \#}$. By the above calculations, $h$ commutes with the differentials. Furthermore we can form a Lie algebra extension $\bar{g}$ of $g$ to $L_{1}^{\prime}\left(k_{1}, n p\right)$ by setting

$$
\bar{g}\left(z^{\prime \prime \#} k_{1}+1\right)= \begin{cases}u\left(\left(k_{1}+1\right) p\right) a_{\left(k_{1}+1\right) p}^{\prime}, & \text { if } k \not \equiv-1(p) ; \\ u\left(\left(k_{1}+1\right) p\right)\left(a_{\left(k_{1}+1\right) p}^{\prime}-p z_{k+1}^{\prime}\right), & \text { if } k \equiv-1(p)\end{cases}
$$

to form a right inverse to $\bar{h}$. Formula (8) implies that this extension commutes with the differentials after reduction modulo $p$. The extra term we have included when $k \equiv-1(p)$ is not essential to the computation, but it makes the determination of the operation of the Bockstein easier. This splitting together with the fact that

$$
d z_{k+1}^{\prime}= \begin{cases}0, & \text { if } k \neq 1(p) \\ c_{\left(k_{1}+1\right) p}^{\prime}, & \text { if } k \equiv-1(p)\end{cases}
$$

shows that the mod- $p$ homology of $\mathcal{U} M_{2}$ is given by

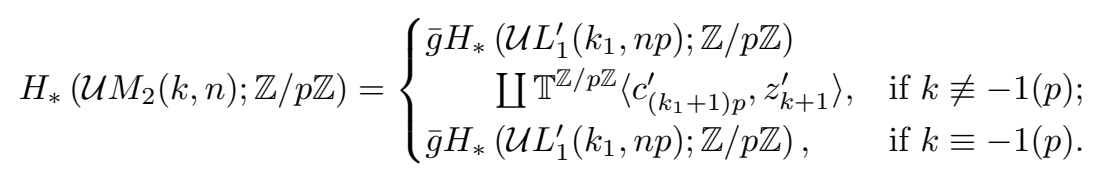

At this point we have

$$
L_{2}=\mathbb{L}\left\langle a_{p^{2}}^{\prime}, y_{p^{2}}, a_{2 p^{2}}^{\prime}, y_{2 p^{2}}, \ldots, a_{k_{2} p^{2}}^{\prime}, y_{k_{2} p^{2}}, z_{k+1}^{\prime}, c_{\left(k_{1}+1\right) p}^{\prime}, a_{\left(k_{1}+1\right) p}^{\prime} \cup C_{2} \cup D_{2}^{\prime}\right\rangle
$$

where $C_{2}$ is as before and $D_{2}^{\prime}$ is a basis for the free $\mathcal{U} \mathbb{L}_{a b}\left\langle z_{p}^{\prime}\right\rangle$-module on

$$
\left\{z_{p}^{\prime} \cdot z_{k+1}^{\prime}, z_{p}^{\prime} \cdot c_{\left(k_{1}+1\right) p}^{\prime}, z_{p}^{\prime} \cdot a_{\left(k_{1}+1\right) p}^{\prime}, b_{k+p+1}\right\} .
$$


As graded Lie algebras, we can rewrite this as

$$
L_{2}=M_{2} \amalg \mathbb{L}\left\langle C_{2}\right\rangle \coprod \mathbb{L}\left\langle D_{2}^{\prime}\right\rangle ;
$$

however, this need not be true as differential graded Lie algebras as $\mathbb{L}\left\langle D_{2}^{\prime}\right\rangle$ might not be closed under the differential. Our final move is to replace $z_{p}^{\prime} \cdot a_{\left(k_{1}+1\right) p}^{\prime}$ by an element $w_{\left(k_{1}+2\right) p}$ so as to rectify this defect.

We consider first the case $k \equiv-1(p)$. In this case $d z_{k+1}^{\prime}=c_{\left(k_{1}+1\right) p}^{\prime}$ and so $d\left(z_{p}^{\prime} \cdot z_{\left(k_{1}+1\right) p}^{\prime}\right)=z_{p}^{\prime} \cdot c_{\left(k_{1}+1\right) p}^{\prime}$. Set $w_{\left(k_{1}+2\right) p}=d b_{k+p+1}$. Explicitly,

$$
\begin{aligned}
& w_{\left(k_{1}+2\right) p}=d b_{k+p+1} \\
& =d\left[y_{p}, z_{k+1}^{\prime}\right] \\
& =p\left[z_{p}^{\prime}, z_{k+1}^{\prime}\right]-\left[y_{p}, c_{\left(k_{1}+1\right) p}^{\prime}\right] \\
& =p\left[z_{p}^{\prime}, z_{k+1}^{\prime}\right]+\left[y_{p}, \frac{d \alpha}{p}\right]+\left[y_{p}, \frac{g d z_{k_{1}+1}^{\#}}{p u\left(\left(k_{1}+1\right) p\right)}\right] \\
& =p\left[z_{p}^{\prime}, z_{k+1}^{\prime}\right] \\
& +\frac{1}{p}\left[y_{p}, d\left(-\frac{1}{2} \sum_{\substack{j=1, j \neq 0(p) \text { or } \\
k_{1}+1-j \neq 0(p)}}^{k_{1}} \frac{1}{p^{t-1}}\left(\begin{array}{c}
\left(k_{1}+1\right) p \\
j p
\end{array}\right)\left[y_{j p}, y_{\left(k_{1}+1\right) p-j p}\right]\right)\right] \\
& +\left[y_{p},\left[L_{2}, L_{2}\right]\right] \\
& =p\left[z_{p}^{\prime}, z_{k+1}^{\prime}\right]-\frac{1}{2 p^{t}}\left[y_{p}, d\left(\left(\begin{array}{c}
\left(k_{1}+1\right) p \\
p
\end{array}\right) 2\left[y_{p}, y_{k_{1} p}\right]\right.\right. \\
& \left.\left.+\sum_{\substack{j=2, j \neq 0(p) \text { or } k_{1}+1-j \neq 0(p)}}^{k_{1}-1}\left(\begin{array}{c}
\left(k_{1}+1\right) p \\
j p
\end{array}\right)\left[y_{j} p, y_{\left(k_{1}+1\right) p-j p}\right]\right)\right]+\left[L_{2}, L_{2}\right] \\
& =p\left[z_{p}^{\prime}, z_{k+1}^{\prime}\right]-\frac{1}{p^{t}}\left(\begin{array}{c}
\left(k_{1}+1\right) p \\
p
\end{array}\right)\left[y_{p}, d\left[y_{p}, y_{k_{1} p}\right]\right]+\left[L_{2}, L_{2}\right] \\
& =p\left[z_{p}^{\prime}, z_{k+1}^{\prime}\right]-\frac{1}{p^{t}}\left(\begin{array}{c}
\left(k_{1}+1\right) p \\
p
\end{array}\right)\left(\left[y_{p},\left[p z_{p}^{\prime}, y_{k_{1} p}\right]\right]-\left[y_{p},\left[y_{p}, d y_{k_{1} p}\right]\right]\right)+\left[L_{2}, L_{2}\right] \\
& =p\left[z_{p}^{\prime}, z_{k+1}^{\prime}\right]-\frac{1}{p^{t}}\left(\begin{array}{c}
\left(k_{1}+1\right) p \\
p
\end{array}\right)\left(\left[p z_{p}^{\prime},\left[y_{p}, y_{k_{1} p}\right]\right]+\left[y_{k_{1} p},\left[y_{p}, p z_{p}^{\prime}\right]\right]\right. \\
& \left.+\frac{1}{2}\left[a_{2 p}, d y_{k_{1} p}\right]\right)+\left[L_{2}, L_{2}\right] \\
& =p\left[z_{p}^{\prime}, z_{k+1}^{\prime}\right]-\frac{1}{p^{t}}\left(\begin{array}{c}
\left(k_{1}+1\right) p \\
p
\end{array}\right)\left(\left[p z_{p}^{\prime}, a_{\left(k_{1}+1\right) p}\right]+\left[y_{k_{1} p}, p b_{2 p}\right]\right)+\left[L_{2}, L_{2}\right] \\
& =p\left[z_{p}^{\prime}, z_{k+1}^{\prime}\right]+\left[z_{p}^{\prime}, a_{\left(k_{1}+1\right) p}^{\prime}\right]+\left[L_{2}, L_{2}\right] .
\end{aligned}
$$

Thus $w_{\left(k_{1}+2\right) p}=d b_{k+p+1}$ is a valid replacement for $z_{p}^{\prime} \cdot a_{\left(k_{1}+1\right) p}^{\prime}$ in this case.

Finally when $k \neq 1(p)$, we will choose $w_{\left(k_{1}+2\right) p}$ in such a way that $d w_{\left(k_{1}+2\right) p}=$ $p z_{p}^{\prime} \cdot c_{\left(k_{1}+1\right) p}$. Since $z_{p}^{\prime} \cdot g d^{\#}{z^{\prime}}_{k_{1}+1}^{\#}$ is a cycle in $\left[z_{p}^{\prime}, K_{2}\right]$, its image in $L_{2}$ is a boundary by Lemma 1.3. Choose $\gamma$ such that $d \gamma=z_{p}^{\prime} \cdot g d^{\#} z_{k_{1}+1}^{\prime \#}$. Since the bracket length 
of $z_{p}^{\prime} \cdot g d^{\#} z_{k_{1}+1}^{\prime \#}$ is greater than 2 , we see that $\gamma \in\left[L_{2}, L_{2}\right]$. Set $w_{\left(k_{1}+2\right) p}=$ $z_{p}^{\prime} \cdot a_{\left(k_{1}+1\right) p}^{\prime}-\gamma / u\left(\left(k_{1}+1\right) p\right)$. Then $w_{\left(k_{1}+2\right) p}$ is a valid replacement for $z_{p}^{\prime} \cdot a_{\left(k_{1}+1\right) p}^{\prime}$ and $d w_{\left(k_{1}+2\right) p}=p z_{p}^{\prime} \cdot c_{\left(k_{1}+1\right) p}^{\prime}$.

Lemma 1.7. For $k \not \equiv-1(p), w_{\left(k_{1}+2\right) p}$ is homologous to $\left[y_{p}, c_{\left(k_{1}+1\right) p}^{\prime}\right]$.

Proof. Write $a \sim b$ to mean $a$ is homologous to $b$. By construction $w_{\left(k_{1}+2\right) p} \sim$ $\left[z_{p}^{\prime}, a_{\left(k_{1}+1\right) p}^{\prime}\right]$. For all $x,\left[y_{p}, d x\right] \sim p\left[z_{p}^{\prime}, x\right]$ since $d\left[y_{p}, x\right]=p\left[z_{p}^{\prime}, x\right]-\left[y_{p}, d x\right]$. Thus for $x \in K_{2},\left[y_{p}, d x\right] \sim\left[z_{p}^{\prime}, x\right] \sim 0$ by Lemma 1.3. By definition $c_{\left(k_{1}+1\right) p}^{\prime}=d a_{\left(k_{1}+1\right) p}^{\prime} / p$ $-d \beta / p^{\nu\left(\left(k_{1}+1\right) p\right)}$ where $\beta \in K_{2}$. Therefore $\left[y_{p}, c_{\left(k_{1}+1\right) p}^{\prime}\right] \sim\left[p z_{p}^{\prime}, a_{\left(k_{1}+1\right) p}^{\prime} / p\right] \sim$ $\left[z_{p}^{\prime}, a_{\left(k_{1}+1\right) p}^{\prime}\right]$.

After this replacement in both cases we get a decomposition as DGL's

$$
L_{2}=M_{2} \amalg C_{2} \amalg N_{2}
$$

where $M_{2}$ and $C_{2}$ are as above and $N_{2}=\mathbb{L}\left\langle D_{2}^{\prime \prime}\right\rangle$ where $D_{2}^{\prime \prime}$ is a basis for the free $\mathcal{U} \mathbb{L}_{a b}\left\langle z_{p}^{\prime}\right\rangle$-module on

$$
\left\{z_{p}^{\prime} \cdot z_{k+1}^{\prime}, z_{p}^{\prime} \cdot c_{\left(k_{1}+1\right) p}^{\prime}, w_{\left(k_{1}+2\right) p}, b_{k+p+1}\right\}
$$

with the differential given by

$$
\left\{\begin{aligned}
d w_{\left(k_{1}+2\right) p} & =p z_{p}^{\prime} \cdot c_{\left(k_{1}+1\right) p}^{\prime} \\
d b_{k+p+1} & =p z_{p}^{\prime} \cdot z_{k+1}^{\prime}
\end{aligned}\right\}, \quad \text { if } k \not \equiv-1(p)
$$

and

$$
\left\{\begin{array}{c}
d z_{p}^{\prime} \cdot z_{k+1}^{\prime}=z_{p}^{\prime} \cdot c_{\left(k_{1}+1\right) p}^{\prime} \\
d b_{k+p+1}=w_{\left(k_{1}+2\right) p}
\end{array}\right\}, \quad \text { if } k \equiv-1(p) .
$$

Applying Lemma 1.2 gives a mod $p$ homology isomorphism

$$
H_{*}\left(\mathcal{U}\left(M_{2} \amalg N_{2}^{\prime}\right) ; \mathbb{Z} / p \mathbb{Z}\right) \rightarrow H_{*}\left(\mathcal{U}\left(M_{2} \amalg N_{2}\right) ; \mathbb{Z} / p \mathbb{Z}\right)
$$

with

$$
N_{2}^{\prime}= \begin{cases}N_{2}, & \text { if } k \not \equiv-1(p) \\ 0, & \text { if } k \equiv-1(p) .\end{cases}
$$

Thus combining this with (10) gives

$$
\begin{aligned}
& H_{*}\left(\mathcal{U} L_{2}^{\prime}(k, n) ; \mathbb{Z} / p \mathbb{Z}\right) \\
& =\left\{\begin{array}{cl}
\bar{g} H_{*}\left(\mathcal{U} L_{1}^{\prime}\left(k_{1}, n p\right) ; \mathbb{Z} / p \mathbb{Z}\right) & \\
\amalg \mathbb{T}^{\mathbb{Z} / p \mathbb{Z}\left\langle\left\{z_{p}^{j} \cdot z_{k+1}^{\prime}\right\}_{j \geq 0},\right.} & \\
\left.\left\{z_{p}^{\prime j} \cdot c_{\left(k_{1}+1\right) p}^{\prime}\right\}_{j \geq 0},\left\{z_{p}^{\prime j} \cdot w_{\left(k_{1}+2\right) p}\right\}_{j \geq 0},\left\{z_{p}^{\prime j} \cdot b_{k+p+1}\right\}_{j \geq 0}\right\rangle, & \text { if } k \not \equiv-1(p) ; \\
\bar{g} H_{*}\left(\mathcal{U} L_{1}^{\prime}\left(k_{1}, n p\right) ; \mathbb{Z} / p \mathbb{Z}\right), & \text { if } k \equiv-1(p)
\end{array}\right.
\end{aligned}
$$

as coalgebras.

The results of the preceding calculations are summarized in the following theorem which determines the coalgebra $H_{*}\left(\Omega J_{k}\left(S^{2 n}\right) ; \mathbb{Z} / p \mathbb{Z}\right)$ in a recursive sense. 
Theorem 1.8. If $k<p$, then $H_{*}\left(\Omega J_{k}\left(S^{2 n}\right) ; \mathbb{Z} / p \mathbb{Z}\right)=\mathbb{S}^{\mathbb{Z} / p \mathbb{Z}}\left\langle y_{1}, z_{k+1}^{\prime}\right\rangle$. For $k \geq$ $p$, as coalgebras

$$
\begin{aligned}
& H_{*}\left(\Omega J_{k}\left(S^{2 n}\right) ; \mathbb{Z} / p \mathbb{Z}\right)
\end{aligned}
$$

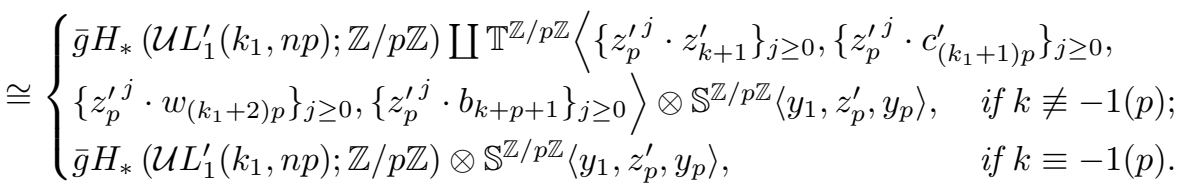

where $H_{*}\left(\mathcal{U} L_{1}^{\prime}(q, m) ; \mathbb{Z} / p \mathbb{Z}\right)=\mathbb{S}^{\mathbb{Z} / p \mathbb{Z}}\left\langle z_{q+1}^{\prime}\right\rangle$ for $q<p$ and

$$
\begin{aligned}
& H_{*}\left(\mathcal{U} L_{1}^{\prime}(q, m) ; \mathbb{Z} / p \mathbb{Z}\right) \cong H_{*}\left(\mathcal{U} L_{2}^{\prime}(q, m) ; \mathbb{Z} / p \mathbb{Z}\right) \otimes \mathbb{S}^{\mathbb{Z} / p \mathbb{Z}}\left\langle z_{p}^{\prime}, y_{p}\right\rangle
\end{aligned}
$$

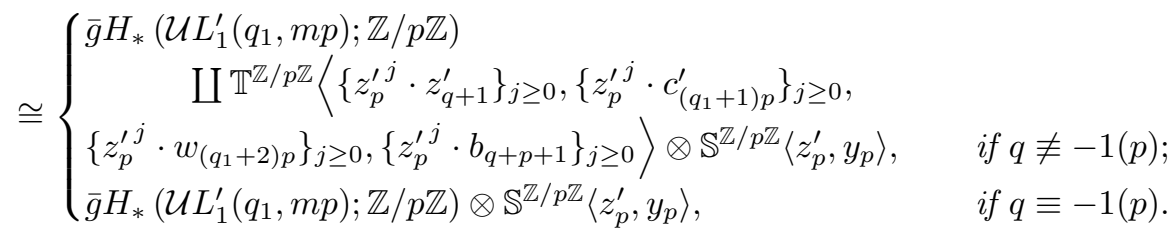

for $q \geq p$.

It is well known (and can be obtained as a limiting case of the preceding calculations) that

$$
H_{*}\left(\Omega J\left(S^{2 n}\right) ; \mathbb{Z} / p \mathbb{Z}\right) \cong \mathbb{S}^{\mathbb{Z} / p \mathbb{Z}}\left\langle v_{2 n-1}, u_{2 n p-2}, v_{2 n p-1}, \ldots, u_{2 n p^{t}-2}, v_{2 n p^{t}-1}, \ldots\right\rangle
$$

where $v_{2 n p^{j}-1}=\bar{g}^{j-1} y_{p, n p^{j-1}}$ and $u_{2 n p^{j}-2}=\bar{g}^{j-1} z_{p, n p^{j-1}}^{\prime}$ for $j \geq 1$, with $v_{2 n-1}=$ $y_{1, n}$. Collecting our earlier results and repeatedly applying the preceding theorem gives the Hopf algebra structure of $H_{*}\left(\Omega J_{k}\left(S^{2 n}\right) ; \mathbb{Z} / p \mathbb{Z}\right)$ described in the following theorem.

Theorem 1.9. If $p^{t} \leq k<p^{t+1}$, then the Hopf algebra $H_{*}\left(\Omega J_{k}\left(S^{2 n}\right) ; \mathbb{Z} / p \mathbb{Z}\right)$ is given by

$$
\begin{aligned}
& H_{*}\left(\Omega J_{k}\left(S^{2 n}\right) ; \mathbb{Z} / p \mathbb{Z}\right) \\
& =\mathbb{T}^{\mathbb{Z} / p \mathbb{Z}}\left\langle\left\{v_{2 n p^{i}-1}\right\}_{i=0}^{t} \cup\left\{u_{2 n p^{i}-2}\right\}_{i=1}^{t}\left\{z_{k_{t}+1, n p^{t}}^{\prime}\right\} \cup\left\{\bar{g}^{t} z_{k_{t}+1, n p^{t}}^{\prime}\right\}\right. \\
& \cup \bigcup_{\substack{i=0 \\
k_{i} \neq=1(p)}}^{t-1}\left(\left\{u_{2 n p^{i+1}-2}^{j} \cdot \bar{g}^{i} z_{k_{i}+1, n p^{i}}^{\prime}\right\}_{j \geq 0} \cup\left\{u_{2 n p^{i+1}-2}^{j} \cdot \bar{g}^{i} c_{\left(k_{i+1}+1\right) p, n p^{i}}^{\prime}\right\}_{j \geq 0}\right. \\
& \left.\left.\cup\left\{u_{2 n p^{i+1}-2}^{j} \cdot \bar{g}^{i} w_{\left(k_{i+1}+2\right) p, n p^{i}}\right\}_{j \geq 0} \cup\left\{u_{2 n p^{i+1}-2}^{j} \cdot \bar{g}^{i} b_{k_{i}+p+1, n p^{i}}\right\}_{j \geq 0}\right)\right\rangle
\end{aligned}
$$

modulo the relations 


$$
\begin{aligned}
& {\left[u_{2 n p^{i}-2}, u_{2 n p^{i^{\prime}-2}}\right]=0, \quad 1 \leq i<i^{\prime} \leq t, \quad 1} \\
& {\left[u_{2 n p^{i}-2}, v_{2 n p^{i^{\prime}}-1}\right]=0, \quad 1 \leq i<i^{\prime} \leq t, \quad 2} \\
& {\left[v_{2 n p^{i}-1}, v_{2 n i^{i^{\prime}-1}}\right]=0, \quad 1 \leq i<i^{\prime} \leq t, \quad 3} \\
& {\left[v_{2 n p^{i+1}-1}, v_{2 n p^{i+1}-1}\right]= \begin{cases}\frac{\bar{g}^{i+1} z_{k_{i+1}+1, n p^{i+1}}^{\prime}}{u(2 p)}, & \text { if } k_{i+1}=2 ; \\
0, & 4\end{cases} } \\
& \begin{array}{ll}
{\left[v_{2 n p^{i+1}-1}, \bar{g}^{i^{\prime}} z_{k_{i^{\prime}}+1, n p^{i^{\prime}}}^{\prime}\right]=0,} & 0 \leq i<i^{\prime}<t, k_{i^{\prime}} \not \equiv-1(p) \text { or } \\
& 0 \leq i<i^{\prime}=t
\end{array} \\
& {\left[v_{2 n p^{i+1}-1}, \bar{g}^{i} z_{k_{i}+1, n p^{i}}^{\prime}\right]=\bar{g}^{i} b_{k_{i}+p+1, n p^{i}}, \quad 0 \leq i<t, k_{i} \not \equiv-1(p), \quad 6} \\
& {\left[v_{2 n p^{i+1}-1}, \bar{g}^{i^{\prime}} c_{\left(k_{i^{\prime}+1}+1\right) p, n p^{i^{\prime}}}^{\prime}\right]=0, \quad 0 \leq i<i^{\prime}<t, k_{i^{\prime}} \not \equiv-1(p), \quad 7} \\
& {\left[v_{2 n p^{i+1}-1}, \bar{g}^{i} c_{\left(k_{i+1}+1\right) p, n p^{i}}^{\prime}\right]=\bar{g}^{i} w_{\left(k_{i+1}+2\right) p, n p^{i}}, \quad 0 \leq i<t, k_{i} \not \equiv-1(p), \quad 8} \\
& {\left[u_{2 n p^{i+1}-2}, \bar{g}^{i^{\prime}} z_{k_{i^{\prime}}+1, n p^{i^{\prime}}}^{\prime}\right]=0, \quad 0 \leq i<i^{\prime}-1<t, k_{i^{\prime}} \not \equiv-1(p), \quad 9} \\
& {\left[u_{2 n p^{i+1}-2}, \bar{g}^{i+1} z_{k_{i+1}+1, n p^{i+1}}^{\prime}\right]=\bar{g}^{i} w_{\left(k_{i+1}+2\right) p, n p^{i}}, \quad 0 \leq i<t, k_{i+1} \not \equiv-1(p), \quad 10} \\
& {\left[u_{2 n p^{i+1}-2}, \bar{g}^{i^{\prime}} c_{\left(k_{i^{\prime}+1}+1\right) p, n p^{i^{\prime}}}^{\prime}\right]=0, \quad 0 \leq i^{\prime}<i<t, k_{i^{\prime}} \not \equiv-1(p), \quad 11} \\
& {\left[v_{2 n-1}, x\right]=\left\{\begin{array}{lr}
z_{k+1}^{\prime}, & \text { if } k=p^{t} \text { and } x=v_{2 n p^{t}-1} \\
0, & \text { if } k \neq p^{t} \text { or } x \text { one of the other } \\
\text { generators. }
\end{array}\right.}
\end{aligned}
$$

Proof. As a coalgebra this follows from repeated application of Theorem 1.8. Turning now to the algebra structure, we must reconstruct the multiplication in the extension $L_{2} \rightarrow L_{1} \rightarrow \mathbb{L}_{a b}\left\langle z_{p}, y_{p}\right\rangle$ and the extension $L_{1} \rightarrow L_{0} \rightarrow \mathbb{L}_{a b}\left\langle y_{1}\right\rangle$. By Lemma 1.3 we have the relations $\left[z_{p}^{\prime}, x\right]=0$ and $\left[y_{p}, x\right]=0$ for

$$
\begin{aligned}
x \in \operatorname{Im}\left(H_{*}\left(\mathcal{U} K_{2}^{\prime}(k, n) ; \mathbb{Z} / p \mathbb{Z}\right)\right. & \cong \bar{g} H_{*}\left(\mathcal{U} K_{1}^{\prime}\left(k_{1}, n p\right) ; \mathbb{Z} / p \mathbb{Z}\right) \\
& \left.\rightarrow \bar{g} H_{*}\left(\mathcal{U} L_{1}^{\prime}\left(k_{1}, n p\right) ; \mathbb{Z} / p \mathbb{Z}\right)\right) .
\end{aligned}
$$

This accounts for relations (1), (2), (3), (5), (7), (9), and (11). Lemma 1.7 gives (8), while (6) and (10) come from the definitions of $b_{k_{i}+p+1}$ and $w_{\left(k_{i+1}+2\right)}$. Relation (4) comes from the fact that $\left[y_{p}, y_{p}\right]=a_{2 p}$ belongs to $I_{2}$ except when $2 p=\left(k_{i}+1\right) p$, in which case $a_{2 p}=\bar{g} z_{2}^{\#} / u(2 p)$ (after reduction modulo $p$ ). Finally the relation $\left[y_{1}, z_{m}^{\prime}\right]=(1 / 2)\left[a_{2}, y_{m-1}\right]$ together with the definition shows $\left[y_{1}, x\right]=0$ for any homology class $x$ except when $k=p^{t}$, in which case $\left[y_{1}, y_{p^{t}}\right]=z_{p^{t}+1}^{\prime}$.

Corollary 1.10. $J_{k}\left(S^{2 n}\right)$ is $\bmod p$ elliptic if and only if $k=q p^{t}-1$ for some $q$ and $t$.

For ease of notation, write $s_{j}$ for $u\left(\left(k_{j}+1\right) p\right)$. Our calculations also give the Bockstein action described in the following theorem.

Theorem 1.11. Suppose $p^{t} \leq k<p^{t+1}$.

$$
\beta\left(v_{2 n p^{j}-1}\right)=u_{2 n p^{j}-2} .
$$


For $i$ such that $k_{i} \not \equiv-1(p)$

$$
\beta\left(\bar{g}^{i} w_{\left(k_{i+1}+2\right) p, n p^{i}}\right)=u_{2 n p^{i+1}-2} \cdot \bar{g}^{i} c_{\left(k_{i+1}+1\right) p, n p^{i}}^{\prime} .
$$

For $i$ such that $i=t$ or $k_{i} \not \equiv-1(p)$

$$
\begin{gathered}
\beta^{(j)}\left(\bar{g}^{i} z_{k_{i}+1, n p^{i}}^{\prime}\right)=0 \quad \text { if } j<i-q \\
\beta^{(i-q)}\left(\bar{g}^{i} z_{k_{i}+1, n p^{i}}^{\prime}\right)=(-1)^{i-q-1} s_{i} s_{i-1} \ldots s_{q+1} \bar{g}^{i-q} c_{k_{q+1}+1, n p^{q}}^{\prime}
\end{gathered}
$$

where $q=\max \left\{\sigma \mid \sigma<i\right.$ and $\left.k_{\sigma} \not \equiv-1(p)\right\}$, and

$$
\beta^{(j)}\left(\bar{g}^{i} z_{k_{i}+1, n p^{i}}^{\prime}\right)=0 \quad \text { for all } j
$$

if there does not exist $\sigma<i$ such that $k_{\sigma} \not \equiv-1(p)$.

For $i$ such that $k_{i} \not \equiv-1(p)$

$$
\beta\left(\bar{g}^{i} b_{k_{i}+p+1, n p^{i}}\right)=u_{2 n p^{i}-2} \cdot \bar{g}^{i} z_{k_{i}+1, n p^{i}}^{\prime}-v_{2 n p^{i+1}-1} \cdot \beta\left(\bar{g}^{i} z_{k_{i}+1, n p^{i}}^{\prime}\right) .
$$

Proof. Since $d \bar{g}=\bar{g} d$ on $K_{1}$, the formulas for $\beta\left(v_{2 n p^{j}-1}\right)$ and $\beta\left(\bar{g}^{i} w_{\left(k_{i+1}+2\right) p, n p^{i}}\right)$ are immediate. However $d$ does not commute with $\bar{g}$ (until after reduction modulo $p$ ) on $z_{k_{i}+1}^{\prime}$ and $b_{k_{i}+p+1}$, so we must do the calculation directly on those elements. The definition, (9), gives for $0 \leq j<i-q$,

$$
\begin{aligned}
d \bar{g}^{j} z_{k_{i}+1, n p^{i}}^{\prime} & =s_{i} d \bar{g}^{j-1} a_{\left(k_{i}+1\right) p, n p^{i}}^{\prime}-p s_{i} d \bar{g}^{j-1} z_{k_{i-1}+1, n p^{i-1}}^{\prime} \\
= & s_{i} d \bar{g}^{j-1} a_{\left(k_{i}+1\right) p, n p^{i-1}}^{\prime}-p s_{i} s_{i-1} d \bar{g}^{j-2} a_{\left(k_{i-1}+1\right) p, n p^{i-2}}^{\prime} \\
& \quad+p^{2} s_{i} s_{i-1} d \bar{g}^{j-2} z_{k_{i-2}+1, n p^{i-2}}^{\prime} \\
= & \ldots \\
= & \sum_{t=0}^{j-1}(-1)^{t} p^{t} s_{i} s_{i-1} \cdots s_{i-t} d \bar{g}^{j-1-t} a_{\left(k_{i-t}+1\right) p, n p^{i-t-1}}^{\prime} \\
& \quad+(-1)^{j} p^{j} s_{i} s_{i-1} \cdots s_{i-j+1} z_{k_{i-j}, n p^{i-j}}^{\prime}
\end{aligned}
$$

and

$$
d \bar{g}^{i-q} z_{k_{i}+1, n p^{i}}^{\prime}=\sum_{t=0}^{i-q-1}(-1)^{t} p^{t} s_{i} s_{i-1} \cdots s_{i-t} d \bar{g}^{i-q-1-t} a_{\left(k_{i-t}+1\right) p, n p^{i-t-1}}^{\prime} .
$$

Since

$$
\begin{aligned}
d \bar{g}^{j-1-t} a_{\left(k_{i-t}+1\right) p, n p^{i-t-1}}^{\prime} & =\bar{g}^{j-1-t} d a_{\left(k_{i-t}+1\right) p, n p^{i-t-1}}^{\prime} \\
& =\bar{g}^{j-1-t}\left(p c_{\left(k_{i-t}+1\right) p, n p^{i-t-1}}^{\prime}+\frac{\bar{g} d z_{k_{i-t}+1, n p^{i-t}}^{\prime}}{s_{i-t}}\right)
\end{aligned}
$$

by (8), and

$$
d z_{k_{m}+1, n p^{m}}^{\prime}= \begin{cases}c_{\left(k_{m+1}+1\right) p, n p^{m}}^{\prime}, & \text { if } i<m<q \\ 0, & \text { if } m=i \text { or } m=q\end{cases}
$$


the sum telescopes to give

$$
d \bar{g}^{j} z_{k_{i}+1, n p^{i}}^{\prime}= \begin{cases}0, & \text { if } j<i-q \\ (-1)^{i-q-1} s_{i} s_{i-1} \ldots s_{q+1} p^{i-q} c_{\left(k_{q+1}+1\right) p, n p^{q}}^{\prime}, & \text { if } j=i-q .\end{cases}
$$

The final formula follows from the definition of $b_{k_{i}+p+1, n p^{i}}$ as $\left[y_{p, n p^{i}}, z_{k_{i}+1, n p^{i}}^{\prime}\right]$.

Notice that the preceding theorem shows that there are some cases in which there is higher $p$-torsion in $H_{*}\left(\Omega J\left(S^{2 n}\right)\right)$, something which does not happen in the limiting case $H_{*}\left(\Omega J\left(S^{2 n}\right)\right)$. Since $\mathcal{P}_{*}^{1} u_{2 n p^{j}-2}=-u_{2 n p^{j-1}-2}^{p}$ in $H_{*}\left(\Omega J\left(S^{2 n}\right) ; \mathbb{Z} / p \mathbb{Z}\right)$, [see CML], this relation also holds in $H_{*}\left(\Omega J_{k}\left(S^{2 n}\right) ; \mathbb{Z} / p \mathbb{Z}\right)$ for $p^{j} \leq k$. The smallest $i$ such that $\bar{g}^{i} z_{k_{i}+1, n p^{i}}^{\prime}$ represents a homology class (i.e. $\max \left\{j \mid k_{\sigma} \equiv-1(p)\right.$ for all $\sigma<j\})$ corresponds to the generator of $H_{2 n(k+1)-2}\left(\Omega J_{k}\left(S^{2 n}\right) ; \mathbb{Q}\right)$.

\section{Product DeCOMPositions And EXPONENTS}

In this section we will produce product decompositions of $\Omega J_{k}\left(S^{2 n}\right)$ for $k<p^{2}-$ $p$ and use it to obtain homotopy exponents for these spaces. The first appearance of a non-trivial Steenrod operation $\mathcal{P}_{*}^{1}$ is in $\Omega J_{p^{2}-p}\left(S^{2 n}\right)$ where $\mathcal{P}_{*}^{1}\left(\bar{g} z_{p, n p}^{\prime}\right)=$ $-\left(u_{2 n p-2}\right)^{p}$. The techniques of this paper are not sufficient to handle that situation. For $p \leq k<p^{2}-p$ we shall obtain a decomposition of the form

$$
\Omega J_{k}\left(S^{2 n}\right) \approx F_{2}(n) \times \Omega S^{2 n(k+1)} \times \Omega \bigvee(\text { Moore spaces })
$$

where $F_{2}(n)$ is a space whose homology is $\mathbb{S}^{\mathbb{Z} / p \mathbb{Z}}\left\langle v_{2 n-1}, u_{2 n p-2}, v_{2 n p-1}\right\rangle$ which was introduced in [S2]. Because of the non-trivial Steenrod operations it is clear that a decomposition of $\Omega J_{k}\left(S^{2 n}\right)$ must involve other types of spaces when $k \geq p^{2}-p$.

It is well known and easy to check that $\Omega J_{k}\left(S^{2 n}\right) \equiv S^{2 n-1} \times \Omega S^{2 n(k+1)-1}$ for $k<p-1$, and $\Omega J_{p-1}\left(S^{2 n}\right)$ is easily seen to be atomic, although it is known to have an exponent from Toda's fibrations. We shall therefore concentrate on the cases $p \leq k<p^{2}-p$. Throughout this section, all homology will be assumed to be with $\mathbb{Z} / p \mathbb{Z}$ coefficients unless stated otherwise.

From section 1 we have

$$
H_{*}\left(\Omega J_{k}\left(S^{2 n}\right)\right)=\mathbb{S}^{\mathbb{Z} / p \mathbb{Z}}\left\langle v_{2 n-1}, u_{2 n p-2}, v_{2 n p-1}\right\rangle \otimes \mathbb{T}^{\mathbb{Z} / p \mathbb{Z}}\langle B\rangle
$$

where

$$
B=\left\{\begin{array}{lr}
\left\{u_{2 n p-2}^{j} \cdot z_{k+1}^{\prime}\right\}_{j \geq 0} \cup\left\{u_{2 n p-2}^{j} \cdot c_{\left(k_{1}+1\right) p}^{\prime}\right\}_{j \geq 0} \\
\cup\left\{u_{2 n p-2}^{j} \cdot w_{\left(k_{1}+2\right) p}\right\}_{j \geq 0} \cup\left\{u_{2 n p-2}^{j} \cdot b_{k+p+1}\right\}_{j \geq 0} \cup\left\{\bar{g} z_{k_{1}+1, n p}^{\prime}\right\}, \\
& \text { if } k \neq \equiv-1(p) \\
\left\{\bar{g} z_{k_{1}+1, n p}^{\prime}\right\}, & \text { if } k \equiv-1(p) .
\end{array}\right.
$$

Since $k<p^{2}-p$ it follows that $k_{1}<p-1$, so the $\gamma$ in the definition of $w_{\left(k_{1}+2\right) p}$ is 0 and thus $w_{\left(k_{1}+2\right) p}=u_{2 n p-2} \cdot a_{\left(k_{1}+1\right) p}^{\prime}$. If $k \not \equiv-1(p)$, then $\bar{g} z_{k_{1}+1, n p}^{\prime}=a_{\left(k_{1}+1\right) p}^{\prime}$, while if $k \equiv-1(p)$, then $\bar{g} z_{k_{1}+1, n p}^{\prime}=u\left(\left(k_{1}+1\right) p\right) a_{\left(k_{1}+1\right) p}^{\prime}-p z_{k+1}^{\prime}$. Thus

$$
B= \begin{cases}\left\{u_{2 n p-2}^{j} \cdot z_{k+1}^{\prime}\right\}_{j \geq 0} \cup\left\{u_{2 n p-2}^{j} \cdot c_{\left(k_{1}+1\right) p}^{\prime}\right\}_{j \geq 0} & \\ \cup\left\{u_{2 n p-2}^{j} \cdot a_{\left(k_{1}+1\right) p}^{\prime}\right\}_{j \geq 0} \cup\left\{u_{2 n p-2}^{j} \cdot b_{k+p+1}\right\}_{j \geq 0}, & \text { if } k \neq-1(p) ; \\ \tilde{a}_{k_{1}+1}, & \text { if } k \equiv-1(p)\end{cases}
$$

where we have used $\tilde{a}_{k_{1}+1}$ to denote $u\left(\left(k_{1}+1\right) p\right) a_{\left(k_{1}+1\right) p}^{\prime}-p z_{k+1}^{\prime}$. 
Our immediate goal is to recall the definition of $F_{2}(n)$, obtain additional properties of it and related spaces, and show that it is a retract of $\Omega J_{k}\left(S^{2 n}\right)$ for $p \leq k<p^{2}-p$.

Lemma 2.1. For each $k$, there exists a map $T_{k}: \Omega J_{k}\left(S^{2 n}\right) \rightarrow \Omega S^{2 n(k+1)-1}$, which is onto on homology.

Proof. This is well known, and there are several possible constructions [Gr], [MN]. One construction, following Toda [T], makes use of the co-action map $J_{k}\left(S^{2 n}\right) \rightarrow$ $J_{k}\left(S^{2 n}\right) \vee S^{2 n k}$ as follows. In general, $\Omega(X \vee Y) \approx \Omega X \times \Omega Y \times \Omega(\Omega X * \Omega Y)$. Define $T_{k}$ to be the composite,

$$
\begin{aligned}
& \Omega J_{k}\left(S^{2 n}\right) \rightarrow \Omega\left(J_{k}\left(S^{2 n}\right) \vee S^{2 n k}\right) \stackrel{\pi_{3}}{\longrightarrow} \Omega\left(\Omega J_{k}\left(S^{2 n}\right) * \Omega S^{2 n k}\right) \\
& \quad \approx \Omega\left(\Sigma \Omega J_{k}\left(S^{2 n}\right) \wedge \Omega S^{2 n k}\right) \rightarrow \Omega\left(\Omega J_{k}\left(S^{2 n}\right) \wedge S^{2 n k}\right) \rightarrow \Omega \Sigma^{2 n k-1} J_{k}\left(S^{2 n}\right) \\
& \quad \approx \Omega\left(\bigvee_{j=1}^{k} S^{2 n k-1+2 n j}\right) \rightarrow \Omega S^{2 n k-1+2 n}=\Omega S^{2 n(k+1)-1} .
\end{aligned}
$$

We refer to such a map as a Toda-Hopf invariant map.

As in [S2], we write $K$ for the $2 n p-2$-skeleton of $\Omega J_{p-1}\left(S^{2 n}\right)$. The following properties of $K$ are shown in [S2].

\section{Lemma 2.2.}

1) $\Sigma^{2} K \approx S^{2 n+1} \vee S^{2 n p}$

2) $\Sigma K \wedge K \approx S^{4 n-1} \vee S^{2 n p+2 n-2} \vee S^{2 n p+2 n-2} \vee S^{4 n p-3}$.

For $0 \leq k \leq p$ let $X_{k}(n)$ denote the homotopy-fibre of the inclusion $J_{k}\left(S^{2 n}\right) \hookrightarrow$ $J_{p-1}\left(S^{2 n}\right)$. From the Serre or Eilenberg-Moore spectral sequence we get

\section{Proposition 2.3.}

$$
H^{*}\left(X_{k}(n)\right)= \begin{cases}\Lambda\left(\tilde{b}_{2 n(k+1)-1}\right) \otimes \Gamma\left(\tilde{u}_{2 n p-2}\right), & \text { if } k<p-1 \\ 0, & \text { if } k=p-1\end{cases}
$$

where $\Lambda(\tilde{b})$ and $\Gamma(\tilde{u})$ denote respectively the exterior algebra on $\tilde{b}$ and the divided polynomial algebra on $\tilde{u}$.

Theorem 2.4. For $1 \leq k<p-1, \Sigma X_{k}(n) \approx \bigvee_{q=1}^{\infty} S^{q(2 n p-2)+1} \bigvee \bigvee_{q=0}^{\infty} S^{q(2 n p-2)+2 n(k+1)}$

Proof. The theorem is equivalent to the statement that the Hurewicz homomorphism $h: \pi_{*}\left(\Sigma X_{k}(n)\right) \rightarrow H_{*}\left(\Sigma X_{k}(n)\right)$ is surjective. For $\epsilon=0$ or 1 , and $q \geq 0$, let $g_{q, \epsilon}$ denote the composite

$$
K^{q} \times\left(S^{2 n(k+1)-1}\right)^{\epsilon} \rightarrow \Omega J_{p-1}\left(S^{2 n}\right) \times X_{k}(n) \stackrel{\mu}{\longrightarrow} X_{k}(n),
$$

where $\mu$ is the action of the fibre on the total space in the principal fibration induced from the fibration defining $X_{k}(n)$. The commutative diagram

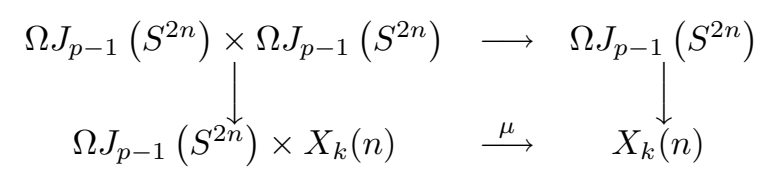


shows that $g_{q, 0_{*}}$ is an isomorphism on $H_{q(2 n p-2)}()$. The commutativity of $\mu_{*}$ with the coproduct then shows that $g_{q, 1_{*}}$ is an isomorphism on $H_{q(2 n p-2)+2 n(k+1)-1}()$. Since $\Sigma(X \times Y) \cong \Sigma X \vee \Sigma Y \vee \Sigma X \wedge Y$, the suspensions of the $g_{k, \epsilon}$ 's can be used in conjuction with the splittings of Lemma 2.2 to produce maps from spheres exhibiting each element of $H_{*}\left(\Sigma X_{k}(n)\right)$ above degree $2 n p-1$ as an image under the Hurewicz homomorphism. Write $B / A$ for the homotopy-theoretic cofibre of $A \rightarrow B$. Let $j: S^{2 n p-2} \rightarrow \Omega J_{p-1}\left(S^{2 n}\right) / S^{2 n-1}$ be a generator of the least nonvanishing homotopy group of that space. Let $c: \Omega J_{p-1}\left(S^{2 n}\right) / \Omega J_{k}\left(S^{2 n}\right) \rightarrow X_{k}(n)$ be induced from the fibration sequence $\Omega J_{k}\left(S^{2 n}\right) \rightarrow \Omega J_{p-1}\left(S^{2 n}\right) \rightarrow X_{k}(n)$. Then the composite

$$
S^{2 n p-2} \stackrel{j}{\longrightarrow} \Omega J_{p-1}\left(S^{2 n}\right) / S^{2 n-1} \rightarrow \Omega J_{p-1}\left(S^{2 n}\right) / \Omega J_{k}\left(S^{2 n}\right) \stackrel{c}{\longrightarrow} X_{k}(n)
$$

induces an isomorphism on $H_{2 n p-2}($ ), and so the the Hurewicz homomorphism is onto in this degree. Finally, the Hurewicz map is an isomorphism in the least non-vanishing degree, $2 n(k+1)-1$, of $X_{k}(n)$.

Remark. In the case $X_{0}(n)=\Omega J_{p-1}\left(S^{2 n}\right)$ the map $c$ does not exist and the above argument gives only the well-known $\Sigma \Omega J_{p-1}\left(S^{2 n}\right) \approx \Sigma K \vee \bigvee_{q=2}^{\infty} S^{q(2 n p-2)+1} \vee$ $\bigvee_{q=1}^{\infty} S^{q(2 n p-2)+2 n p}$ as in $[\mathrm{S} 2]$.

Corollary 2.5. For $1 \leq k \leq p-1, X_{k}(n) \approx S^{2 n(k+1)-1} \times \Omega S^{2 n p-1}$.

Proof. Using adjoints of maps coming from the preceding wedge decomposition, we get $\operatorname{maps}_{k}(n) \rightarrow \Omega S^{2 n p-1}$ and $X_{k} \rightarrow \Omega S^{2 n(k+1)} \approx S^{2 n(k+1)-1} \times \Omega S^{4 n(k+1)-1} \rightarrow$ $S^{2 n(k+1)-1}$ which are onto on homology. Together they give a map $X_{k}(n) \rightarrow$ $S^{2 n(k+1)-1} \times \Omega S^{2 n p-1}$ which induces a homology isomorphism and thus is a homotopy equivalence.

For degree reasons, the restriction to $J_{q p^{t}-1}\left(S^{2 n}\right)$ of the $p^{t}$ th Hopf invariant map $H: J\left(S^{2 n}\right) \rightarrow J\left(S^{2 n p^{t}}\right)$ lands in $J_{q-1}\left(S^{2 n p^{t}}\right)$, the $2 n p^{t}(q-1)$-skeleton of $J\left(S^{2 n p^{t}}\right)$. Note that in the notation of section 1 , if $k_{0}=q p-1$, then $k_{1}=q-1$. The map $H: J_{q p-1}\left(S^{2 n}\right) \rightarrow J_{q-1}\left(S^{2 n p}\right)$ induces the map $h: K_{2}\left(k_{0}, n\right) \rightarrow K_{1}\left(k_{1}, n p\right)$ of section 1 .

Theorem 2.6. $J_{p^{t}-1}\left(S^{2 n}\right) \hookrightarrow J_{q p^{t}-1}\left(S^{2 n}\right) \stackrel{H}{\longrightarrow} J_{q-1}\left(S^{2 n p^{t}}\right)$ is a fibration sequence up to homotopy.

Proof. The proof is the same as that of the existence of Toda's homotopy-fibration $J_{p-1}\left(S^{2 n}\right) \rightarrow J\left(S^{2 n}\right) \rightarrow J\left(S^{2 n p}\right)$. Toda's calculation [T] shows that $H$ induces an isomorphism on $\mathbb{Z}_{(p)}$-cohomology in degrees $2 n j p^{t}$ and consequently exhibits $H^{*}\left(J_{q p^{t}-1}\left(S^{2 n}\right) ; \mathbb{Z}_{(p)}\right)$ as a free module over $H^{*}\left(J_{q-1}\left(S^{2 n p^{t}}\right) ; \mathbb{Z}_{(p)}\right)$ with a basis for $H^{*}\left(J_{p^{t}-1}\left(S^{2 n}\right) ; \mathbb{Z}_{(p)}\right)$ as basis. The Serre or Eilenberg-Moore spectral sequence thus shows that the homology of the homotopy-fibre of $H$ is isomorphic to that of $J_{p^{t}-1}\left(S^{2 n}\right)$. The composite $J_{p^{t}-1}\left(S^{2 n}\right) \rightarrow J_{q p^{t}-1}\left(S^{2 n}\right) \rightarrow J_{q-1}\left(S^{2 n p^{t}}\right)$ is trivial for degree reasons and so yields a map from $J_{p^{t}-1}\left(S^{2 n}\right)$ to the homotopy-fibre of $H$ which induces the homology isomorphism above. 
These Hopf invariant maps are compatible in the sense that if $q \leq q^{\prime}$, then

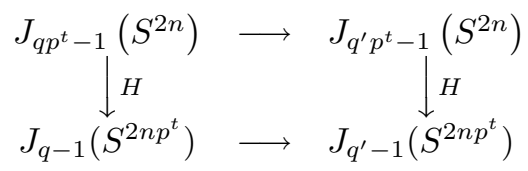

commutes, and the preceding theorem shows that this square is a homotopytheoretic pullback. In particular, the homotopy-fibre of $J_{q p-1}\left(S^{2 n}\right) \rightarrow J_{p^{2}-1}\left(S^{2 n}\right)$ is $X_{q-1}(n p)$, the same as that of $J_{q-1}\left(S^{2 n p}\right) \rightarrow J_{p-1}\left(S^{2 n p}\right)$.

For $1 \leq q<p$, let $j_{q}$ denote the composite $S^{2 n p q-1} \rightarrow X_{q-1}(n p) \rightarrow J_{q p-1}\left(S^{2 n}\right)$. In particular, $j_{1}$ is the composite $S^{2 n p-1} \rightarrow \Omega J_{p-1} S^{2 n p} \stackrel{\partial}{\longrightarrow} J_{p-1}\left(S^{2 n}\right)$ where the second map is induced from the fibration $J_{p-1}\left(S^{2 n}\right) \rightarrow J_{p^{2}-1}\left(S^{2 n}\right) \rightarrow J_{p-1}\left(S^{2 n p}\right)$.

\section{Lemma 2.7.}

1) For $1 \leq q<p, j_{q}$ is the attaching map by means of which $J_{q p}\left(S^{2 n}\right)$ is constructed from $J_{q p-1}\left(S^{2 n}\right)$. In other words, $S^{2 n p q-1} \stackrel{j_{q}}{\longrightarrow} J_{q p-1}\left(S^{2 n}\right) \rightarrow J_{q p}\left(S^{2 n}\right)$ is a cofibration sequence up to homotopy.

2) For $2 \leq q<p, \Omega j_{q}$ induces an injection on $H_{*}($ ).

Proof. 1) Given a fibration $F \rightarrow E \rightarrow B$, Ganea [Ga] shows that the homotopyfibre of the induced map $E / F \rightarrow B$ is $F * \Omega B$. The homotopy fibration $X_{q-1}(n p) \rightarrow$ $J_{q p-1}\left(S^{2 n}\right) \rightarrow J_{p^{2}-1}\left(S^{2 n}\right)$ thus yields a homotopy fibration

$$
X_{q-1}(n p) * \Omega J_{p^{2}-1}\left(S^{2 n}\right) \rightarrow J_{q p-1}\left(S^{2 n}\right) / X_{q-1}(n p) \rightarrow J_{p^{2}-1}\left(S^{2 n}\right)
$$

which shows that $J_{q p-1}\left(S^{2 n}\right) / X_{q-1}(n p) \rightarrow J_{p^{2}-1}\left(S^{2 n}\right)$ is more than $2 n p q$ connected. Since $S^{2 n p q-1} \rightarrow X_{q-1}(n p)$ is also more than $2 n p q$-connected, the composite

$$
J_{q p-1}\left(S^{2 n}\right) / S^{2 n p q-1} \rightarrow J_{q p-1}\left(S^{2 n}\right) / X_{q-1}(n p) \rightarrow J_{p^{2}-1}\left(S^{2 n}\right)
$$

is $2 n p q$-connected, and so the homotopy cofibre of $j_{q}$ is $J_{p q}\left(S^{2 n}\right)$, the $2 n p q$-skeleton of $J_{p^{2}-1}\left(S^{2 n}\right)$.

2) Looping before applying Ganea shows by the above argument that the composite

$$
\Omega J_{q p-1}\left(S^{2 n}\right) / \Omega S^{2 n p q-1} \rightarrow \Omega J_{q p-1}\left(S^{2 n}\right) / \Omega X_{q-1}(n p) \rightarrow \Omega J_{p^{2}-1}\left(S^{2 n}\right)
$$

is $2 n p q$-connected. Since $q>1$, we know

$$
H_{*}\left(\Omega J_{q p-1}\left(S^{2 n}\right)\right)=\mathbb{S}^{\mathbb{Z} / p \mathbb{Z}}\left\langle v_{2 n-1}, u_{2 n p-2}, v_{2 n p-1}\right\rangle \otimes \mathbb{T}^{\mathbb{Z} / p \mathbb{Z}}\left\langle\tilde{a}_{q p}\right\rangle,
$$

where $\left|\tilde{a}_{q p}\right|=2 n p q-2$. However

$$
H_{*}\left(\Omega J_{p^{2}-1}\left(S^{2 n}\right)\right)=\mathbb{S}^{\mathbb{Z} / p \mathbb{Z}}\left\langle v_{2 n-1}, u_{2 n p-2}, v_{2 n p-1}, u_{2 n p^{2}-2}\right\rangle
$$

so this map can be $2 n p q$ connected only if $\Omega j_{q_{*}}$ is an injection in degree $2 n p q-2$. Since it is multiplicative, this means that it is an injection in all degrees.

The space $F_{2}(n)$ was defined in [S2] as the homotopy-theoretic pullback of the maps $\Omega H: \Omega J\left(S^{2 n}\right) \rightarrow \Omega J\left(S^{2 n p}\right)$ and $E^{2}: S^{2 n p-1} \rightarrow \Omega^{2} S^{2 n p+1} \approx \Omega J\left(S^{2 n p}\right)$. Since each of these maps is an $H$-map, this description makes it clear that $F_{2}(n)$ is an 
$H$-space. The diagram

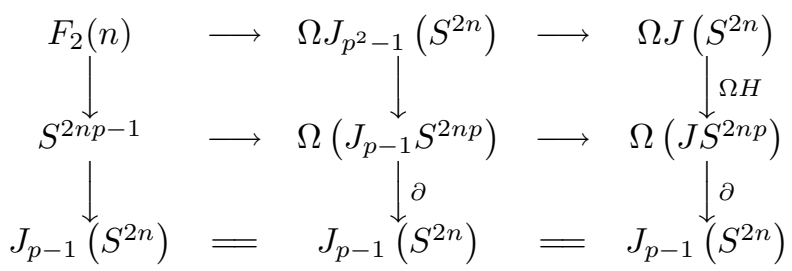

in which the top squares are homotopy-theoretic pullbacks and the columns are fibrations up to homotopy shows that $F_{2}(n)$ is also the homotopy fibre of the composite $S^{2 n p-1} \rightarrow \Omega\left(J_{p-1} S^{2 n p}\right) \stackrel{\partial}{\longrightarrow} J_{p-1}\left(S^{2 n}\right)$ which was earlier identified as $j_{1}$, the attaching map for the formation of $J_{p}\left(S^{2 n}\right)$.

The diagram of homotopy fibrations

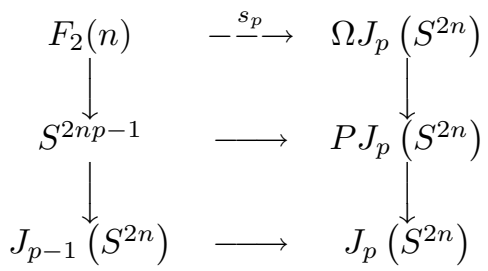

produces an induced map $s_{p}$ between the homotopy fibres. Although the homotopy class of $s_{p}$ is not uniquely determined by the diagram (but depends on exactly how the homotopy commutative diagram is realized as a strictly commuting diagram of fibrations), any choice of $s_{p}$ makes the diagram

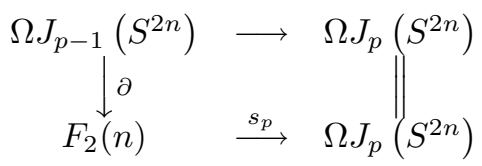

homotopy commute and so induces an isomorphism on $H_{2 n-1}()$ and $H_{2 n p-2}()$. For $k \geq p$ let $s_{k}$ denote the composite $F_{2}(n) \rightarrow \Omega J_{p}\left(S^{2 n}\right) \rightarrow \Omega J_{k}\left(S^{2 n}\right)$. We will construct a left homotopy inverse to $s_{k}$ for $p \leq k<p^{2}-p$ by producing a left homotopy inverse to $s_{p^{2}-p-1}$.

\section{Lemma 2.8.}

1) For $1 \leq q<p$ the homotopy fibre of $j_{q}: S^{2 n p q-1} \rightarrow J_{q p-1}\left(S^{2 n}\right)$ is $F_{2}(n)$.

2) For $p \leq k<p^{2}-p, s_{k}: F_{2}(n) \rightarrow \Omega J_{k}\left(S^{2 n}\right)$ has a left homotopy inverse $r_{k}$.

Proof. 1) We have already shown this for $q=1$, so suppose $q>1$. Let $F$ be homotopy fibre of $j_{q}$. We know that $H_{*}\left(\Omega J_{q p-1}\left(S^{2 n}\right)\right)=H_{*}\left(F_{2}(n)\right) \otimes H_{*}\left(\Omega S^{2 n p q-1}\right)$ and that $\Omega j_{q}$ induces an injection on homology. From this we can calculate that $H_{*}(F)$ equals $H_{*}\left(F_{2}(n)\right)$. The composite $F_{2}(n) \stackrel{s_{q p-1}}{\longrightarrow} \Omega J_{q p-1}\left(S^{2 n}\right) \stackrel{\partial}{\longrightarrow} F$ induces an isomorphism on homology in degrees $2 n-1$ and $2 n p-2$ and so is a homotopy equivalence by the "atomicity" style lemma which follows.

2) In part 1 this was shown for integers of the form $q p-1$ where $2 \leq q<p$, and so in particular for $p^{2}-p-1$. Therefore for all $k$ the composite

$$
\Omega J_{k}\left(S^{2 n}\right) \rightarrow \Omega J_{p^{2}-p-1}\left(S^{2 n}\right) \stackrel{r_{p^{2}-p-1}}{\longrightarrow} F_{2}(n)
$$

is a left homotopy inverse to $s_{k}$. 
Lemma 2.9. Suppose that $F$ and $G$ are simply connected spaces such that $H_{*}(F) \cong$ $H_{*}(G) \cong H_{*}\left(F_{2}(n)\right)$ as co-algebras. Let $f: F \rightarrow G$ be a map which induces an isomorphism on homology in degrees $2 n-1$ and $2 n p-2$. Then $f$ is a homotopy equivalence.

Proof. Write $a, u$, and $v$ for the algebra generators of $H_{*}\left(F_{2}(n)\right)$ in degrees $2 n-1$, $2 n p-2$ and $2 n p-1$ respectively. Commutativity of $f_{*}$ with the coproduct shows that the least degree in which $f_{*}$ fails to be an isomorphism must contain a primitive. $P H_{*}\left(F_{2}(n)\right)=\left\langle a, v, u, u^{p}, \ldots, u^{p^{k}}, \ldots\right\rangle$. By hypothesis, $f_{*}(a) \neq 0$ and $f_{*}(u) \neq 0$. Commutativity with the Bockstein shows that $f_{*}(v) \neq 0$. Suppose by induction that $f_{*}\left(u^{p^{m}}\right) \neq 0$ for $m \leq k$. Then commutativity of $f_{*}$ with the coproduct shows that $f_{*}\left(u^{p^{k}} v\right) \neq 0$ since there are no primitives in its degree. Applying $\beta$ then shows that $f_{*}\left(u^{p^{k+1}}\right) \neq 0$ to complete the induction. Thus $f_{*}$ is an injection on primitives and so is an isomorphism. Therefore $f$ is a homotopy equivalence.

The final thing we need in order to produce our homotopy decomposition of $\Omega J_{k}\left(S^{2 n}\right)$ for $p \leq k<p^{2}-p$ is to show that certain elements in $H_{*}\left(\Omega J_{k}\left(S^{2 n}\right)\right)$ are in the image of the mod $p$ Hurewicz homomorphism.

Lemma 2.10. Suppose $k \geq p$ and let $u$ denote the generator of $H_{*}\left(\Omega J_{k}\left(S^{2 n}\right)\right)$ in degree $2 n p-2$. If $x \in H_{*}\left(\Omega J_{k}\left(S^{2 n}\right)\right)$ lies in the image of the mod $p$ Hurewicz homomorphism, then so does $[u, x]$.

Proof. Let [, ] : $\Omega J_{k}\left(S^{2 n}\right) \wedge \Omega J_{k}\left(S^{2 n}\right) \rightarrow \Omega J_{k}\left(S^{2 n}\right)$ be the Samelson product. Let $i$ be the composite $K \rightarrow \Omega J_{p-1}\left(S^{2 n}\right) \rightarrow \Omega J_{k}\left(S^{2 n}\right)$. Suppose $x \in H_{*}\left(\Omega J_{k}\left(S^{2 n}\right)\right)$ is the Hurewicz image of $f: P^{N}(p) \rightarrow J_{k}\left(S^{2 n}\right)$. Then $[u, x]$ lies in the image of the map induced on homology by the composite $K \wedge P^{N}(p) \stackrel{i \wedge f}{\longrightarrow} \Omega J_{k}\left(S^{2 n}\right) \wedge$ $\Omega J_{k}\left(S^{2 n}\right) \stackrel{[,]}{\longrightarrow} \Omega J_{k}\left(S^{2 n}\right)$. However by Lemma $2.2, K \wedge P^{N}(p) \cong P^{N+2 n-1}(p) \vee$ $P^{N+2 n p-2}(p)$, and so $[u, x]$ lies in the image of the $\bmod p$ Hurewicz homomorphism.

Theorem 2.11. For $k$ such that $p \leq k<p^{2}-p$ and $k \not \equiv-1(p)$, the elements $z_{k+1}^{\prime}$, $a_{\left(k_{1}+1\right) p}^{\prime}$, and $b_{k+p+1}^{\prime}$ of $H_{*}\left(\Omega J_{k}\left(S^{2 n}\right)\right)$ lie in the image of the mod $p$ Hurewicz homomorphism.

Proof. Write simply $z, a$, and $b$ for $z_{k+1}^{\prime}, a_{\left(k_{1}+1\right) p}^{\prime}$, and $b_{k+p+1}^{\prime}$ respectively. Let $G$ be the homotopy fibre of $r_{k}: \Omega J_{k}\left(S^{2 n}\right) \rightarrow F_{2}(n)$. $z$ is the Hurewicz image of the composite $P^{2 n(k+1)}(p) \rightarrow S^{2 n(k+1)} \stackrel{j}{\longrightarrow} G \rightarrow \Omega J_{k}\left(S^{2 n}\right)$, where $j$ is a generator of the least nonvanishing homotopy group of $G$. This composite will henceforth be written as $\tau$. Let $G^{\prime}$ be the homotopy-theoretic fibre of the composite $G \rightarrow$ $\Omega J_{k}\left(S^{2 n}\right) \stackrel{T_{k}}{\longrightarrow} \Omega S^{2 n(k+1)-1}$. There is a map $P^{2 n p q-2}(p) \rightarrow G^{\prime}$ which on homology hits the two least nonvanishing degrees of $H_{*}\left(G^{\prime}\right)$. The Hurewicz image of the composite $P^{2 n p q-2}(p) \rightarrow G^{\prime} \rightarrow G \rightarrow \Omega J_{p}\left(S^{2 n}\right)$ is $a$. Finally, writing $L$ for the $2 n p-1$ skeleton of $F_{2}(n)$, we have from [S2] that $\Sigma^{2} L \approx S^{2 n+1} \vee P^{2 n p+1}(p)$. Since $b$ lies in the image of the map induced on homology by the composite $P^{2 n(k+1)} \wedge$ $L \stackrel{\tau \wedge()}{\longrightarrow} \Omega J_{k}\left(S^{2 n}\right) \wedge \Omega J_{k}\left(S^{2 n}\right) \stackrel{[,]}{\longrightarrow} \Omega J_{k}\left(S^{2 n}\right)$, the wedge decomposition of $\Sigma^{2} L$ shows that $b$ lies in the image of the $\bmod p$ Hurewicz homomorphism. 
Similarly,

Theorem 2.12. For $k$ such that $p \leq k<p^{2}-p$ and $k \equiv-1(p)$, the element $\tilde{a}_{k+1}$ lies in the image of the mod $p$ Hurewicz homomorphism.'

Theorem 2.13. For $k=q p+r$ such that $p \leq k<p^{2}-p$ and $k \not \equiv-1(p)$

$$
\begin{aligned}
& \Omega J_{k}\left(S^{2 n}\right) \approx F_{2}(n) \\
& \times \Omega\left(S^{2 n(k+1)-1} \vee \bigvee_{j=0}^{\infty} P^{2 n(q+1) p+j(2 n p-2)-1}(p) \vee \bigvee_{j=0}^{\infty} P^{2 n(k+p+1)+j(2 n p-2)-2}(p)\right) .
\end{aligned}
$$

Proof. By Theorem 2.11 and Lemma 2.10, we can find maps $S^{2 n(k+1)-2} \rightarrow$ $\Omega J_{k}\left(S^{2 n}\right), P^{2 n(q+1) p+j(2 n p-2)-1}(p) \rightarrow \Omega J_{k}\left(S^{2 n}\right)$, and $P^{2 n(k+p+1)+j(2 n p-2)-2}(p)$ $\rightarrow \Omega J_{k}\left(S^{2 n}\right)$ whose Hurewicz images are $z, a d^{k}(u)(a)$, and $a d^{k}(u)(b)$ respectively. Since $\Omega J_{k}\left(S^{2 n}\right)$ is an $H$-space, these maps yield a map

$$
\begin{aligned}
& \phi: \Omega\left(S^{2 n(k+1)-1} \vee \bigvee_{j=0}^{\infty} P^{2 n(q+1) p+j(2 n p-2)-1}(p) \vee \bigvee_{j=0}^{\infty} P^{2 n(k+p+1)+j(2 n p-2)-2}(p)\right) \\
& \rightarrow \Omega J_{k}\left(S^{2 n}\right)
\end{aligned}
$$

by the universal property of the James construction. By construction the composite

$$
\begin{aligned}
F_{2}(n) \times \Omega\left(S^{2 n(k+1)-1}\right. & \vee \bigvee_{j=0}^{\infty} P^{2 n(q+1) p+j(2 n p-2)-1}(p) \\
& \left.\vee \bigvee_{j=0}^{\infty} P^{2 n(k+p+1)+j(2 n p-2)-2}(p)\right) \\
& \stackrel{s_{k} \times \phi}{\longrightarrow} \Omega J_{k}\left(S^{2 n}\right) \times \Omega J_{k}\left(S^{2 n}\right) \rightarrow \Omega J_{k}\left(S^{2 n}\right)
\end{aligned}
$$

induces a homology isomorphism and is thus a homotopy equivalence.

Similarly

Theorem 2.14. For $k$ such that $p \leq k<p^{2}-p$ and $k \equiv-1(p)$

$$
\Omega J_{k}\left(S^{2 n}\right) \approx F_{2}(n) \times \Omega S^{2 n(k+1)-1} .
$$

Applying the Hilton-Milnor theorem and the main theorems of [CMN3] and [N3] gives

Corollary. $J_{k}\left(S^{2 n}\right)$ has a homotopy exponent for $k<p^{2}-p$.

\section{REFERENCES}

[AH] J.F. Adams and P. Hilton, On the chain algebra of a loop space, Comment. Math. Helv. 30 (1955), 305-330. MR 17:11196

[A] D. Anick, Differential algebras in topology, Research Notes in Math. 3, A.K. Peters Ltd., 1993. MR 94h:55020

[CML] F. Cohen, T. Lada, P. May, Homology of iterated loop spaces, Springer Lecture Notes in Math. Vol. 533, Springer-Verlag, 1976. MR 55:9096 
[CMN1] F. Cohen, J. Moore, J. Neisendorfer, Torsion in the homotopy groups, Ann. of Math. 109 (1979), 121-168. MR 80e:55024

[CMN2] F. Cohen, J. Moore, J. Neisendorfer, The double suspension and exponents in the homotopy groups of spheres, Ann. of Math. 110 (1979), 121-168. MR 81e:55021

[CMN3] F. Cohen, J. Moore, J. Neisendorfer, Exponents in homotopy theory, Algebraic Topology and K-Theory, Ann. of Math. Studies 113 (1987), 3-34. MR 89d:55035

[FHT] Y. Felix, S. Halperin, J.-C. Thomas, The homotopy Lie algebra for finite complexes, Publ. Math. I.H.E.S. 56 (1982), 387-410. MR 85e:55010

[Ga] T. Ganea, A generalization of the homology and homotopy suspension, Comment. Math. Helv 39 (1965), 295-322. MR 31:4033

[Gr] B. Gray, On Toda's fibration, Math. Proc. Camb. Phil. Soc. 97 (1985), 289-298. MR 86i: 55016

$[\mathrm{H}] \quad$ P. Hilton, On the homotopy groups of the union of spheres, J. London Math. Soc. 30 (1955), 154-172. MR 16:847d

[J] I. James, Reduced product spaces, Ann. of Math. 62 (1955), 170-197. MR 17:3966

$[\mathrm{MN}] \quad$ J. Moore and J. Neisendorfer, Equivalence of Toda-Hopf invariants, Israel J. Math. 66 (1-3) (1989), 300-318. MR 90g:55013

[N1] J. Neisendorfer, Primary homotopy theory, Mem. AMS No. 232, 1980. MR 81b:55035

[N2] J. Neisendorfer, The exponent of a Moore space, Algebraic Topology and K-Theory, Ann. of Math. Studies 113 (1987), 35-71. MR 89e:55029

[N3] J. Neisendorfer, 3-primary exponents, Math. Proc. Camb. Phil. Soc. 90 (1981), 63-83. MR 82e:55026

[NS] J. Neisendorfer and P. Selick, Some examples of spaces with or without exponents, Modern Trends in Algebraic Topology II, Can. Math. Soc. Proc. 2 (1982), 343-357. MR 84b:55017

[S1] P. Selick, Odd primary torsion in $\pi_{k}\left(S^{3}\right)$, Topology 17 (1978), 407-412. MR 80c:55010

[S2] P. Selick, A spectral sequence concerning the double suspension, Invent. Math. 64 (1981), 15-24. MR 82j:55015

[S3] P. Selick, Moore conjectures, Alg. Top. - Rational Homotopy, Springer Lecture Notes in Math. 1318, 1988, pp. (219-227). MR 90c:55014

[S4] P. Selick, Constructing product filtrations by means of a generalization of a theorem of Ganea, Trans. Amer. Math. Soc. 348 (1996), 3573-3589. CMP 95:12

[T] H. Toda, On the double suspension $E^{2}$, J. Institute Polytech. Osaka City Univ., Ser. A 7 (1956), 103-145. MR 19:1188g

Department of Mathematics, University of Toronto, Toronto, Ontario, Canada M5S $1 \mathrm{~A} 1$

E-mail address: selick@math.toronto.edu 